\title{
Handling Parameter Variations during the Treatment of Type 1 Diabetes Mellitus: In Silico Results
}

\author{
María F. Villa Tamayo $\mathbb{D}{ }^{1},{ }^{1}$ Michelle A. Caicedo Alvarez $\mathbb{D}{ }^{1},{ }^{1}$ and Pablo S. Rivadeneira $\mathbb{D}{ }^{1,2}$ \\ ${ }^{1}$ Universidad Nacional de Colombia, Facultad de Minas, Grupo GITA, Cra. 80\# 65-223, Medellín, Colombia \\ ${ }^{2}$ Instituto de Desarrollo Tecnológico para la Industria Química INTEC - CONICET, Santa Fe 3000, Argentina \\ Correspondence should be addressed to Pablo S. Rivadeneira; psrivade@unal.edu.co
}

Received 15 October 2018; Revised 5 February 2019; Accepted 27 March 2019; Published 28 May 2019

Academic Editor: Pasquale Palumbo

Copyright (c) 2019 María F. Villa Tamayo et al. This is an open access article distributed under the Creative Commons Attribution License, which permits unrestricted use, distribution, and reproduction in any medium, provided the original work is properly cited.

Type 1 diabetic patients need a strict treatment to regulate blood glucose concentration in a target range. Despite the development of different control strategies, the model parameter variations, given by physiological changes, can generate an inaccurate treatment and in consequence hyperglycemia and hypoglycemia episodes. Therefore, it is necessary to use control techniques that compensate such effects and maintain the control goals. Here, the effect of parametric variations is examined by the sensitivity analysis from which the most influential parameters in glycemia dynamics are detected. Based on that, an offset-free MPC strategy for impulsive systems is given for the first time in literature and simulated for type 1 diabetes treatment. This scheme along with the impulsive zone MPC with artificial variables reestablishes the normoglycemia behavior since the parameter variations are adequately rejected. However, only parametric variations up to $50 \%$ from their nominal values are well compensated, which suggests that more robust formulations are needed to ensure a greater rejection of physiological variations.

\section{Introduction}

Type 1 diabetes mellitus (T1DM) is an autoimmune disease in which the pancreatic $\beta$-cells are destroyed, causing inability to secrete insulin and regulate blood glucose (BG) in the body. Additionally, it leads to secondary pathophysiological alterations in many systems and can be the cause of nephropathy, blindness, or even nontraumatic amputations of lower extremities [1]. To counteract this dysfunction, one of the most common treatments is functional insulin therapy. It consists of daily insulin injections according to glycemia measurements and carbohydrate intake with the objective of maintaining normoglycemia $(70 \mathrm{mg} / \mathrm{dl} \leq \mathrm{BG} \leq 180 \mathrm{mg} / \mathrm{dl})$. Another treatment option is based on the development of the project called Artificial Pancreas (AP). AP attempts to emulate the natural behavior of the pancreas by the use of an insulin pump, continuous glucose monitoring, and closed-loop control strategies. This system aims to avoid hypoglycemia $(\mathrm{BG}<70 \mathrm{mg} / \mathrm{dl})$ and hyperglycemia $(\mathrm{BG}>180$ $\mathrm{mg} / \mathrm{dl}$ ) events, which result in complications in the patient with T1DM [2].
AP has been extensively studied the last decade, and several control strategies have been proposed to close the loop, ranging from PID up to more complex strategies such as model predictive control. Recently, Medtronic has released to the market the first commercial device using a closedloop strategy producing astonishing results. It consists of a proportional-integral-derivative with insulin feedback of a model-predicted insulin profile (PID-IFB) with safety constraints, to calculate the basal insulin delivery, and a manual option for bolus insulin [3]. This indicates that it is necessary to maintain the effort to get fully automated strategies. In this regard, one of the most studied control strategies is precisely model predictive control (MPC), which has shown adequate performances in clinical and in silico environments [4-6].

The prediction capacity of the MPC strategy provides the selection of the optimal insulin administration that drives glycemia to the objective according to a cost function. Additionally, the zone MPC (ZMPC), which allows BG regulation in the target zone without requiring an extra correction to take it to a set-point, has shown a significant reduction of hypoglycemia and hyperglycemia events [7]. 
Recently, an incursion of impulsive control strategies applied to biomedical systems has been done. Due to the characteristics of T1DM treatment, the input can be approximated as an impulse when the insulin bolus is injected because of its short duration in relation to the sample time [8]. Many biomedical applications, such as HIV, malaria, and diabetes, are better modeled and controlled as impulsive systems [8]. In [9], an initial application of impulsive ZMPC (iZMPC) to T1DM patient dynamics is introduced. Furthermore, an iZMPC with artificial variables (iZMPC-AV) is developed in [10], where the use of these variables guarantees the convergence to an objective set and provides an enlarged domain of attraction.

An essential component in MPC is the prediction model that describes the interaction between glycemia, insulin, and carbohydrate absorption. Several models have been developed: maximal models which seek a detailed representation of a diabetic patient and the control-oriented models. The most used model for control is the Bergman minimal model [11], which has been modified to include different features such as meal absorption [12]. Based on this results, advances as the improvement of the UVa/Padova simulator [13] and the implementation with control strategies as MPC [14] have been done. Recently, many control-oriented models have been introduced; in $[15,16]$, different long-term models are presented, with a validation of 2 and 12 days, respectively. These have realistic equilibriums and are simple due to their affine linear forms. Finally, in [17, 18], a modification of the last two models is developed, considering different time constants in the compartments of absorption of insulin and carbohydrates and the endogenous glucose. The model in [18] is used in this paper.

So far, control techniques assume that the model adequately represents the process dynamics, which is not completely true due to the inherent mismatch implicated by the variability on human physiology caused by physical activity, hormonal changes, stress, the dawn phenomenon, and other factors. As a consequence of this assumption, when the resulting control is applied to the patient, hypoglycemia or hyperglycemia events may occur. With the purpose of understanding the plant-model mismatch, here, a sensitivity analysis is carried out as explained in [19] to determine the parameters that have more influence in glycemia. Subsequently, tests are conducted with different variation scenarios of the parameters.

To overcome the inconvenience of the mismatch evidenced in the tests, the offset-free MPC strategy developed in $[20,21]$ is adopted. Here, an extension of this strategy is performed for impulsive systems for the first time in literature. To assess the performance of the impulsive MPC strategies, a large comparison using six different formulations is developed. In conclusion, the iZMPC-AV has the best performance. Based on that, this strategy is adapted to the offset-free scheme. The adaptation consists of an augmented system with a disturbance model and a state estimator (the Kalman estimator is chosen). The information provided by the estimator is used by the MPC control to achieve the target zone and, in clinical terms, to reduce the possibility of hypoglycemia and hyperglycemia episodes, even when there are parametric variations.
The outline of the paper is as follows: In the Methodology, a description of the selected model for glucose-insulincarbohydrate dynamics is presented. Then, a subsection is devoted to introduce the iZMPC-AV formulation. In addition, a sensitivity analysis of the closed-loop system is addressed to determine the parameters that have more influence in glycemia. The section concludes with the description of the offset-free control strategy. In the Results and Discussion, a performance comparison between the MPC strategies for discrete systems and the MPC strategies for impulsive systems, applied to T1DM treatment problems, is carried out. Afterwards, the issue shown by the mismatch is illustrated by the sensitivity analysis, and the results and performance evaluation of the offset-free control strategy are presented. Perspectives and conclusions are discussed at the end.

\section{Methodology}

The development of the offset-free MPC strategy applied to T1DM treatment consists of four fundamental subsections: the model to describe the interaction between glucose, insulin, and carbohydrates; the appropriate MPC formulation to maintain glycemia in the target zone; the sensitivity analysis of the influence of the parameters in the glycemia dynamics; and an extended MPC formulation considering the offset-free problem.

2.1. Glucose-Insulin-Carbohydrate Model. Different models have been developed to describe the glucose-insulincarbohydrate interaction. Among them, the ones presented in $[15,16]$ are advantageous for having a long-term validity, and their equilibriums adequately describe the physiology of a patient with type 1 diabetes. Two recent models are those introduced in $[17,18]$. Both models present a great similarity, differentiating themselves from the previously mentioned, mainly by including a term associated with glucose selfregulation mechanism, which allows the glucose level to be driven toward a basal state, stabilizing the system, and by using different time constants for each compartment of absorption of insulin. In this work, the selected model is the one in [18], since it also allows the use of different time constants for each compartment of absorption of carbohydrates. The model is formed by five state variables, and its state space representation is

$$
\begin{gathered}
\dot{x}(t)=A x(t)+B_{u} u(t)+B_{r} r(t)+E, \\
A=\left[\begin{array}{ccccc}
-p_{0} & -p_{1} & 0 & p_{2} & 0 \\
0 & -\frac{1}{p_{4}} & \frac{1}{p_{5}} & 0 & 0 \\
0 & 0 & -\frac{1}{p_{6}} & 0 & 0 \\
0 & 0 & 0 & -\frac{1}{p_{8}} & \frac{1}{p_{9}} \\
0 & 0 & 0 & 0 & -\frac{1}{p_{11}}
\end{array}\right],
\end{gathered}
$$


TABLE 1: Parameters description of the model.

\begin{tabular}{lcc}
\hline Parameter & Description & Units \\
\hline$p_{0}$ & Fractional rate of glucose self-regulation. & $1 / \mathrm{min}$ \\
$p_{1}$ & Insulin action effectiveness. & $\mathrm{mg} / \mathrm{U} / \mathrm{min}$ \\
$p_{2}$ & Carbohydrate bioavailability. & - \\
$p_{3}$ & Net balance between endogenous glucose production and insulin independent glucose consumption. & $\mathrm{mg} / \mathrm{dl} / \mathrm{min}$ \\
$p_{4}, p_{6}$ & Diffusion time constants in the insulin compartments. & $\mathrm{min}$ \\
$p_{5}, p_{7}$ & Insulin diffusion effectiveness in each compartment. & - \\
$p_{8}, p_{10}$ & Diffusion time constants in the two digestion compartments. & min \\
$p_{9}, p_{11}$ & Meal absorption effectiveness in each compartment. & - \\
\hline
\end{tabular}

$$
B_{u}=\left[\begin{array}{c}
0 \\
0 \\
\frac{1}{p_{7}} \\
0 \\
0
\end{array}\right], B_{r}=\left[\begin{array}{c}
0 \\
0 \\
0 \\
0 \\
\frac{1}{p_{10}}
\end{array}\right], E=\left[\begin{array}{c}
p_{3} \\
0 \\
0 \\
0 \\
0
\end{array}\right]
$$

The five state variables are as follows: $x_{1}$, the glycemia-blood glucose concentration $(\mathrm{mg} / \mathrm{dl}) ; x_{2}$ and $x_{3}$, the delivery rates of insulin in the blood and interstitial space compartments, respectively ( $\mathrm{U} / \mathrm{min}$ ); and $x_{4}$ and $x_{5}$, the delivery rates of carbohydrates in the stomach and duodenum compartments, respectively $(\mathrm{g} / \mathrm{min})$. The inputs are $u$, the exogenous insulin $(\mathrm{U})$, and $r$, the intake of carbohydrates due to meals $(\mathrm{g})$. The output of the system is the glycemia, i.e., $y=\left[\begin{array}{lllll}1 & 0 & 0 & 0 & 0\end{array}\right] x$.

The initial conditions and model parameters were identified for the 33 virtual patients from the UVa/Padova simulator [13] and also validated with clinical data of 42 real patients. The identification algorithm and details can be seen in [18]. One important feature is that all parameters have physiological meaning; their description and units are exhibited in Table 1.

\subsection{Impulsive Zone Model Predictive Control with Artificial} Variables Strategy. A recent approach for the control of the system is by considering the input of insulin as an impulse. Insulin doses are administered as small spaced pulses, rather than a continuous input or a discrete one. Then, the consideration of it as an impulsive system is appropriate to emulate the real T1DM treatment $[9,22]$. Affine linear impulsive systems are described as follows:

$$
\begin{aligned}
\dot{x}(t) & =A x(t)+B_{r} r(t)+E, \quad x(0)=x_{0}, \quad t \neq \tau_{k}, \\
x\left(\tau_{k}^{+}\right) & =x\left(\tau_{k}\right)+B_{u} u\left(\tau_{k}\right), \quad k \in \mathbb{N},
\end{aligned}
$$

where $x \in X \subseteq \mathbb{R}^{n}$ is the constrained state vector, $u \in$ $U \subseteq \mathbb{R}^{m}$ denotes the constrained impulsive control input, and $r \subseteq \mathbb{R}^{m_{r}}$ denotes the disturbances due to meals. Both $X$ and $U$ are compact sets and contain the origin. The impulsive-time instants are denoted by $\tau_{k}, k \in \mathbb{N}$, and $\tau_{k}^{+}$refers to the time instant after $\tau_{k}$. As explained in [23], from the relationship between the state after the free response $x\left(\tau_{k}\right)$ and the state after the jump $x\left(\tau_{k}^{+}\right)$given by the application of the impulsive input, it is possible to expand the characterization of (2) by means of two underlying discrete-time subsystems at time instants $\tau_{k}$ and $\tau_{k}^{+}$, respectively:

$$
\begin{aligned}
& x^{\bullet}(k+1)=A^{\bullet} x^{\bullet}(k)+B_{u^{*}}^{*}(k)+B_{r^{*}}^{r^{*}}(k)+E^{*}, \\
& x^{\bullet}(0)=x\left(\tau_{0}\right), \\
& x^{\circ}(k+1)=A^{\circ} x^{\circ}(k)+B_{u}^{\circ} u^{\circ}(k)+B_{r}^{\circ} r^{\circ}(k)+E^{\circ}, \\
& x^{\circ}(0)=x\left(\tau_{0}^{+}\right),
\end{aligned}
$$

where $A^{\bullet}=e^{A T}, A^{\circ}=e^{A T}, B_{u}^{\circ}=e^{A T} B_{u}, B_{u}^{\circ}=B_{u}, B_{r}^{\bullet}=$ $B_{r}^{\circ}=\int_{0}^{T} e^{A s} d s B_{r}, E^{\bullet}=e^{A T} E, E^{\circ}=E, u^{\circ}(k)=u^{\bullet}(k)$, and $T=\tau_{k+1}-\tau_{k}$ is a constant period of impulses. The state $x\left(\tau_{k}\right)$ is denoted as $x^{\circ}(k)$, and $x\left(\tau_{k}^{+}\right)$is $x^{\circ}(k)$. The purpose is to control the whole system by the manipulation of (3).

Afterward, the control objective is to drive the linear impulsive system to a nonempty target set $X_{s}^{\cdot T a r} \subseteq X_{s} \subseteq X$, with its associated input target set $U_{s}^{\text {Tar }} \subseteq U_{s} \subseteq U$, where $X_{s}$ and $U_{s}$ are the equilibrium sets of the system and maintain the system inside $X_{s}^{\cdot T a r}$ indefinitely. Note that the equilibrium control is not applied continuously as it happens in discretetime systems since now the control system is impulsive, and it cannot be applied in this form. The equilibrium control is also sent impulsively at each time $\tau_{k}$, which leads to a generalized equilibrium. This concept was studied at [10] including its dynamical characterization.

All MPC algorithms have three essential elements for their formulation: the prediction model needed to compute the predicted output, a cost function in a prediction horizon $N$, and the constraints associated with the nature of the system [24-26]. The iZMPC-AV formulation was developed and substantiated in [10]. Here, that formulation is applied to the T1DM treatment problem since there is a required zone to maintain glycemia, and the iZMPC-AV is a stable formulation. This strategy is chosen due to its performance in comparison to other MPC formulations for discrete and impulsive systems, as it will be shown later. The performance comparison of these formulations is established in the Results and Discussion. 
Denote $X_{s}^{\bullet} \subseteq X_{s}$ as the equilibrium set to the subsystem (3) and its associated set of equilibrium inputs $U_{s}$. The cost function of the iZMPC-AV is then

$$
\begin{aligned}
& V_{N}\left(\dot{x}^{\bullet}, r^{\bullet}, X_{s}^{\cdot T a r}, U_{s}^{\text {Tar }} ; \boldsymbol{u}, u_{a}, x_{a}\right) \\
& \quad=V_{d y n}\left(x^{\bullet}, \dot{r} ; \boldsymbol{u}, x_{a}, u_{a}\right)+V_{f}\left(X_{s}^{\cdot T a r}, U_{s}^{\text {Tar }} ; x_{a}, u_{a}\right)
\end{aligned}
$$

where $V_{d y n}$ and $V_{f}$ are

$$
\begin{aligned}
& V_{d y n}\left(x^{\bullet}, r^{\bullet} ; \boldsymbol{u}, x_{a}, u_{a}\right) \\
& =\sum_{i=0}^{N-1}\left\|x^{\bullet}(k+i \mid k)-x_{a}\right\|_{Q}^{2} \\
& \quad+\sum_{i=0}^{N-1}\left\|u^{\bullet}(k+i \mid k)-u_{a}\right\|_{R}^{2} \\
& V_{f}\left(X_{s}^{\cdot T a r}, U_{s}^{\text {Tar }} ; x_{a}, u_{a}\right) \\
& =P\left(\text { dist }_{X_{s}^{\cdot T a r}}\left(x_{a}\right)+\text { dist }_{U_{s}^{\text {Tar }}}\left(u_{a}\right)\right),
\end{aligned}
$$

with $Q, R, P$ being semidefinite positive matrices. The term $V_{d y n}$ is meant to steer the system to a certain artificial equilibrium given by $\left(u_{a}, x_{a}\right) \in U_{s} \times X_{s}^{*}$. The term $V_{f}$ is a terminal cost function; it represents the deviation between the artificial equilibrium point and any other point $\left(u_{t}, x_{t}\right)$ in the objective equilibrium set $\left(U_{s}^{\text {Tar }}, X_{s}^{\bullet T a r}\right)$.

The optimization problem to be solved by the MPC at time $k$ is

$$
\begin{array}{ll}
\min _{\boldsymbol{u}, u_{a}, x_{a}} & V_{N}\left(x^{\bullet}, r^{\bullet}, X_{s}^{\cdot T a r}, U_{s}^{\text {Tar }} ; \boldsymbol{u}, u_{a}, x_{a}\right) \\
\text { s.t. } & x^{\bullet}(0)=x^{\bullet}(k \mid k), \\
& x^{\bullet}(k+i \mid k) \\
& =A^{\bullet} x^{\bullet}(k+i-1 \mid k)+B_{u^{\bullet}}^{\bullet}(k+i-1 \mid k) \\
& +B_{r}^{\bullet} r^{\bullet}(k+i-1 \mid k)+E^{\bullet}, \\
& y(k+i \mid k)=C x^{\bullet}(k+i \mid k), \\
& u^{\bullet}(k+i \mid k) \in U, \\
& x^{\bullet}(k+i \mid k) \in X, \\
& x^{\bullet}(k+N \mid k)=x_{a}, \\
& x_{a}=A^{\bullet} x_{a}+B_{u^{\bullet}} u_{a}+E^{\bullet}, \quad\left(x_{a} \in X_{s}^{\bullet}, u_{a} \in U_{s}\right)
\end{array}
$$

where the constraint $x^{\bullet}(k+N \mid k)=x_{a}$ drives the terminal state at the end of the horizon to reach the artificial equilibrium $x_{a}$, and the constraint $x_{a}=A^{\bullet} x_{a}+B_{u}^{\bullet} u_{a}+E^{\bullet}$ forces the pair $\left(u_{a}, x_{a}\right)$ to fulfill the equilibrium condition. Notice that the information about the disturbance due to the meals, $B_{r}^{\bullet} r^{\bullet}(\bullet)$, is entered into the formulation, but the control strategy will assume that the intake of meals is unannounced.
2.3. Sensitivity Analysis. To expose the dependence of $x$ to the parameters and their variations, a sensitivity analysis is performed. This denotes which of the parameters are the most influential in the change of blood glucose, for example. Assuming the identified parameters as the nominal value $p_{n}$ of the parameters $p$, the sensitivity function is defined [19]:

$$
\begin{aligned}
S_{x_{i}, p_{j}} & =\frac{\partial x_{i}}{\partial p_{j}}, \\
\dot{S}_{x_{i}, p_{j}}(t) & =A S_{x_{i}, p_{j}}(t)+\frac{\partial f\left(x, u, p_{n}\right)}{\partial p}, \quad S_{x_{i}, p_{j}}(0)=0,
\end{aligned}
$$

where $S_{x, p}$ represents the sensitivity of the state $x$ to the parameters $p=\left[p_{0} p_{2} \ldots p_{11}\right]$ of model 2.1. Then, the analysis of variations of the most influential parameters is done to retrieve information about the effect on the control performance when there is a plant-model mismatch.

2.4. The Offset-Free iZMPC-AV Strategy. To compensate the plant-model mismatch given by variation of parameters, the offset-free MPC strategy introduced in $[20,21]$ is adapted here for the first time to the impulsive formulation stated in Section 2.2. The idea of the strategy is to have some knowledge about the mismatch and provide this information to the iZMPC-AV. For that purpose, the affine impulsive system (2) is augmented with an additional model of the disturbance to capture the plant-model mismatch in the steady state. It is assumed that the perturbation is constant, i.e., $\dot{d}(t)=0$, and it affects the state in the impulse instants. Then, the augmented system has the form

$$
\begin{gathered}
{\left[\begin{array}{c}
\dot{x}(t) \\
\dot{d}(t)
\end{array}\right]=\left[\begin{array}{cc}
A & 0 \\
0 & 0
\end{array}\right]\left[\begin{array}{l}
x(t) \\
d(t)
\end{array}\right]+\left[\begin{array}{c}
B_{r} \\
0
\end{array}\right] r(t)+\left[\begin{array}{c}
E \\
0
\end{array}\right],\left[\begin{array}{c}
x(0) \\
d(0)
\end{array}\right]=\left[\begin{array}{c}
x_{0} \\
0
\end{array}\right], t \neq \tau_{k},} \\
x\left(\tau_{k}^{+}\right)=x\left(\tau_{k}\right)+B_{u} u\left(\tau_{k}\right)+B_{d} d\left(\tau_{k}\right), \quad k \in \mathbb{N} \\
y\left(\tau_{k}\right)=C x\left(\tau_{k}\right)+C_{d} d\left(\tau_{k}\right)
\end{gathered}
$$

in which $d \in \mathbb{R}^{n_{d}}$ is the constant disturbance, and its associated matrices are $B_{d} \in \mathbb{R}^{n_{x} \times n_{d}}$ and $C_{d} \in \mathbb{R}^{n_{y} \times n_{d}}$. Therefore, the underlying discrete system described in (3) and the output equation are rewritten as

$$
\begin{aligned}
\tilde{x}^{\bullet}(k+1)= & \widetilde{A}^{\bullet} \tilde{x}^{\bullet}(k)+\widetilde{B}_{u^{\bullet}} u^{\bullet}(k)+\widetilde{B}_{r}^{\bullet} r^{\bullet}(k)+\widetilde{E}^{\bullet} \\
& +w(k), \quad \widetilde{x}^{\bullet}(0)=\widetilde{x}\left(\tau_{0}\right), \\
\tilde{y}(k)= & \widetilde{C} \tilde{x}^{\bullet}(k)+v(k),
\end{aligned}
$$

where $\tilde{x}^{\bullet}(k)=\left[x^{\bullet}(k) d^{\bullet}(k)\right]^{\prime}$ denotes the augmented state, $\tilde{y}$ is the augmented output, and the augmented matrices of the new system (12) are given by $\widetilde{A}=\left[\begin{array}{cc}A^{\bullet} & B_{d}^{*} \\ 0 & I\end{array}\right], \widetilde{B}_{u}^{\bullet}=\left[\begin{array}{c}\widetilde{B}_{u}^{*} \\ 0\end{array}\right]$, $\widetilde{B}_{r}^{*}=\left[\begin{array}{c}\widetilde{B}_{r}^{*} \\ 0\end{array}\right], \widetilde{E}^{\bullet}=\left[\begin{array}{c}E^{*} \\ 0\end{array}\right], \widetilde{C}=\left[\begin{array}{ll}C & C_{d}\end{array}\right]$, and $B_{d}^{\bullet}=e^{A T} B_{d}$. Additionally, the term $w(k)$ denotes a zero-mean and $Q_{e^{-}}$ covariance Gaussian process noise entering into the states, and $v(k)$ is a zero-mean and $R_{e}$-covariance Gaussian process noise affecting the measure of the system. Afterward, the condition in which the state and the disturbance can be 
estimated is that the pair $(\widetilde{C}, \widetilde{A})$ is observable. It is satisfied if and only if

$$
\operatorname{rank}\left[\begin{array}{c}
\widetilde{C} \\
\widetilde{C} \widetilde{A} \cdot \\
\vdots \\
\widetilde{C} \widetilde{A}^{\cdot n_{x}+n_{d}-1}
\end{array}\right]=n_{x}+n_{d} \text {, }
$$

and, then, the state and disturbance estimator is given by

$$
\begin{aligned}
\widetilde{\tilde{x}}^{\bullet}(k+1)= & \widetilde{A} \cdot \widetilde{\widetilde{x}}(k)+\widetilde{B}_{u}^{\cdot} \cdot \dot{u}(k)+\widetilde{B}_{r}^{\cdot} \cdot{ }^{\bullet}(k)+\widetilde{E} \\
& +K_{e}(\tilde{y}(k)-\widetilde{C} \widetilde{\widetilde{x}}(k)),
\end{aligned}
$$

in which the gain matrix $K_{e}=\left[\begin{array}{ll}K_{1} & K_{2}\end{array}\right]^{\prime}$ is chosen so that the estimator is stable. The selected method to estimate the augmented state is the Kalman estimator [27]. This estimator consists of a prediction or a priori stage (15) denoted by $\tilde{\tilde{x}}^{--}$, which is an estimate of $\tilde{x}^{\cdot}$ before the measurement $\tilde{y}$ is taken into account, and a correction stage or a posteriori stage (16) denoted by $\widehat{\widetilde{x}}^{\bullet+}$, which is the estimate of $\tilde{x}^{\bullet}$ after the measurement is processed. $P_{e}$ denotes the covariance of the estimation error, $K_{e}$ is the Kalman filter gain, and $R_{e}$ and $Q_{e}$ are the covariance matrices associated with measurement noise and model noise, respectively.

$$
\begin{aligned}
& \widehat{\widetilde{x}}^{\cdot-}(k+1)=\widetilde{A} \cdot \widehat{\widetilde{x}}^{\cdot+}(k)+\widetilde{B}_{u}^{\cdot} u(k)+\widetilde{B}_{r}^{\cdot} r^{\cdot}(k)+\widetilde{E}, \\
& P_{e}^{-}(k+1)=\widetilde{A} P_{e}^{+}(k) \widetilde{A}^{\cdot T}+Q_{e}, \\
& K_{e}(k+1)=P_{e}^{-}(k+1) \widetilde{C}^{\prime}\left(\widetilde{C} P_{e}^{-}(k+1) \widetilde{C}^{\prime}+R_{e}\right)^{-1}, \\
& \widehat{\widetilde{x}}^{\cdot+}(k+1) \\
& =\tilde{\widetilde{x}}^{\cdot-}(k+1) \\
& \quad+K_{e}(k+1)\left(\widetilde{y}(k+1)-\widetilde{C} \widetilde{\tilde{x}}^{\cdot-}(k+1)\right), \\
& P_{e}^{+}(k+1) \\
& =\left(I-K_{e}(k+1) \widetilde{C}\right) P_{e}^{-}(k+1)\left(I-K_{e}(k+1) \widetilde{C}\right)^{\prime} \\
& \quad+K_{e}(k+1) R_{e} K_{e}^{\prime}(k+1)
\end{aligned}
$$

Assuming that the estimator is stable and the number of disturbances is equal to the number of outputs, in steady state, it satisfies

$$
\left[\begin{array}{cc}
A^{\bullet}-I & B_{u}^{\cdot} \\
C & 0
\end{array}\right]\left[\begin{array}{l}
\widehat{x}_{\infty}^{\cdot} \\
u_{\infty}^{\cdot}
\end{array}\right]=\left[\begin{array}{c}
-E^{\bullet}-B_{d}^{\cdot} \widehat{d}_{\infty} \\
y_{\infty}-C_{d} \widehat{d}_{\infty}
\end{array}\right],
$$

where the subscript $\infty$ denotes the steady state values. Given the current augmented state $\ddot{\widetilde{x}}(k)$, the optimization problem coupled with the observation problem to solve in each time step $k$ is

$$
\begin{aligned}
& \min _{\boldsymbol{u}, u_{a}, x_{a}} V_{N}\left(\tilde{x}^{\bullet}, r^{\bullet}, X_{s}^{\cdot T a r}, U_{s}^{\text {Tar }} ; \boldsymbol{u}, u_{a}, x_{a}\right) \\
& \text { s.t. } \tilde{x}^{\bullet}(0)=\widetilde{\widetilde{x}}^{\bullet}(k \mid k) \text {, } \\
& \tilde{x}^{*}(k+i \mid k) \\
& =\widetilde{A}^{*} \tilde{x}^{*}(k+i-1 \mid k)+\widetilde{B}_{u}^{*} \cdot u^{\bullet}(k+i-1 \mid k) \\
& +\widetilde{B}_{r}^{\bullet} r^{\bullet}(k+i-1 \mid k)+\widetilde{E}^{\bullet}, \\
& \tilde{y}(k+i \mid k)=\widetilde{C} \widetilde{x}(k+i \mid k), \\
& u \cdot(k+i \mid k) \in U \text {, } \\
& x^{*}(k+i \mid k) \in X, \\
& x^{\bullet}(k+N \mid k)=x_{a}, \\
& x_{a}=A^{\bullet} x_{a}+B_{u^{*}}^{*} u_{a}+B_{d}^{\bullet} d+E^{\bullet} \text {, } \\
& \left(x_{a} \in X_{s}^{\bullet}, u_{a} \in U_{s}\right), \\
& y_{a}=C x_{a}+C_{d} d(k+i \mid k),
\end{aligned}
$$

where $V_{N}\left(\widetilde{x}^{\bullet}, r^{\bullet}, X_{s}^{\cdot T a r}, U_{s}^{\text {Tar }} ; \boldsymbol{u}, u_{a}, x_{a}\right)$ is the augmented version of the cost function in (5)-(7) and the difference between the plant and the model is included in the calculation of the artificial equilibrium $x_{a}$. Let $y_{a, \infty}$ be the artificial reference in steady state. To assure that the variables will have zero offset, the steady state values must satisfy the observer condition (17) and the artificial values must satisfy the equilibrium constraint of the controller.

$$
\left[\begin{array}{cc}
A^{\bullet}-I & B_{u}^{*} \\
C & 0
\end{array}\right]\left[\begin{array}{l}
x_{a, \infty} \\
u_{a, \infty}
\end{array}\right]=\left[\begin{array}{l}
-E^{\bullet}-B_{d}^{*} \widehat{d}_{\infty} \\
y_{a, \infty}-C_{d} \widehat{d}_{\infty}
\end{array}\right]
$$

In (18) the terms $x_{a}=A^{*} x_{a}+B_{u^{*}} u_{a}+B_{d}^{*} d+E^{*}$ and $y_{a}=$ $C x_{a}+C_{d} d(k+i \mid k)$ refer to this equilibrium constraint of the controller. Note that, as in the nominal MPC formulation (see Section 2.2), the intake of meals is available information for the control strategy, but it will not be used since a secondary objective of the control is to be able to counteract the unknown disturbances, and then the meals will be considered as unannounced.

\section{Results and Discussion}

3.1. Glycemia Control in T1DM Patients: Comparison of Six MPC Strategies. In this subsection, different MPC strategies for discrete and impulsive systems, applied to the T1DM treatment problem, are compared. These strategies are the standard discrete MPC (dMPC) [24], the discrete ZMPC (dZMPC) with slack variable [26], the discrete ZMPC with artificial variables (dZMPC-AV) [28, 29], and the adaptation of the same formulations for impulsive systems: iMPC [30], iZMPC [22], and iZMPC-AV [10]; the description of each formulation can be seen in the appendix. 
TABLE 2: Performance comparison of the MPC strategies for discrete and impulsive systems. Values are medians (interquartile range) unless stated otherwise.

\begin{tabular}{|c|c|c|c|c|c|c|}
\hline Strategy & iMPC & dMPC & iZMPC & dZMPC & $\begin{array}{c}\text { iZMPC- } \\
\text { AV }\end{array}$ & $\begin{array}{c}\text { dZMPC- } \\
\text { AV }\end{array}$ \\
\hline Mean (SD) BG (mg/dl) & $127.8(10.7)$ & $121.6(12.2)$ & $129.2(4.9)$ & $125.5(8.8)$ & $112.5(8.7)$ & $112.3(8.4)$ \\
\hline $\mathrm{SD}(\mathrm{SD})(\mathrm{mg} / \mathrm{dl})$ & $36.2(12.7)$ & $30.9(11.9)$ & $27.9(12.1)$ & $31.7(10.9)$ & $27.5(13.0)$ & $29.0(12.8)$ \\
\hline \multicolumn{7}{|c|}{ Time percentage of BG (\%) } \\
\hline$<70 \mathrm{mg} / \mathrm{dl}$ & $0(0)$ & $0(0)$ & $0(0)$ & $0(0)$ & $0(0)$ & $0(0)$ \\
\hline$\in[70,140] \mathrm{mg} / \mathrm{dl}$ & $73.2(11.4)$ & $76.9(11.9)$ & $74.1(7.8)$ & $75.2(11.3)$ & $83.9(11.8)$ & $83.8(12.8)$ \\
\hline$\in[70,180] \mathrm{mg} / \mathrm{dl}$ & $91.5(14.6)$ & $94.9(14.8)$ & $94.9(9.5)$ & $93.9(11.4)$ & $98.7(7.9)$ & $97.8(9.3)$ \\
\hline$>180 \mathrm{mg} / \mathrm{dl}$ & $8.5(14.6)$ & $5.1(14.8)$ & $5.0(9.5)$ & $6.1(11.4)$ & $1.3(7.9)$ & $2.2(9.3)$ \\
\hline$>250 \mathrm{mg} / \mathrm{dl}$ & $0(2.0)$ & $0(1.1)$ & $0(0)$ & $0(1.6)$ & $0(0)$ & $0(0)$ \\
\hline$>300 \mathrm{mg} / \mathrm{dl}$ & $0(0)$ & $0(0)$ & $0(0)$ & $0(0)$ & $0(0)$ & $0(0)$ \\
\hline \multicolumn{7}{|l|}{ Number of events BG (-) } \\
\hline$<70 \mathrm{mg} / \mathrm{dl}$ & $0(0)$ & $0(0)$ & $0(0)$ & $0(0)$ & $0(0)$ & $0(0)$ \\
\hline$>180 \mathrm{mg} / \mathrm{dl}$ & $2(2)$ & $2(3)$ & $1(2)$ & $1(2)$ & $1(2)$ & $1(2)$ \\
\hline$>250 \mathrm{mg} / \mathrm{dl}$ & $0(1)$ & $0(1)$ & $0(0)$ & $0(1)$ & $0(0)$ & $0(0)$ \\
\hline$>300 \mathrm{mg} / \mathrm{dl}$ & $0(0)$ & $0(0)$ & $0(0)$ & $0(0)$ & $0(0)$ & $0(0)$ \\
\hline
\end{tabular}

The three mentioned techniques for discrete and impulsive systems are applied using model 2.1 and assuming that the plant and the model are equal, i.e., the obtained results are from nominal conditions. As stated before, the formulation of each of the control strategies does not consider the announcement of meals; instead these are considered as unknown disturbances. Therefore, the term $B_{r} r(t)$ is not considered in the prediction model. The simulated scenario is based on 33 virtual patients from the UVa/Padova simulator, which has 10 children, 10 adolescents, and 10 adults with their average patients, respectively. The initial condition for $x_{1}$ is set in $120 \mathrm{mg} / \mathrm{dl}$. The objective is to maintain the system in the target zone, even when food intake is entered into the system. The time of the simulation is 24 hours, and meals considered are $50 \mathrm{~g}$ of breakfast at 7:00h, lunch of $80 \mathrm{~g}$ at $12: 00 \mathrm{~h}$, and dinner of $60 \mathrm{~g}$ at $21: 00 \mathrm{~h}$. The prediction horizon $N$ and the matrices $Q, R, P$ are adjusted for each patient using the Nelder-Mead method for autotuning to maximize the time inside the target zone [31]. This tuning procedure is appropriate for in silico environments but other strategies have to be considered for use in real-life conditions such as the one presented in [32]. To all patients, the constraint sets are $\{0,0,0\} \leq X \leq\{1000,10,10\}$ and $0 \leq U \leq 30$. In addition, the reference for all strategies is set in $100 \mathrm{mg} / \mathrm{dl}$. The target zone of the glycemia is set as $80 \leq x_{1} \leq 140$ for all controllers, and the zone called normoglycemia is defined as $70 \leq x_{1} \leq 180$. As detailed in [33], a value above that range is considered as level 1 hyperglycemia and a value below it as level 1 hypoglycemia, and the critical events $x_{1}<54 \mathrm{mg} / \mathrm{dl}$ and $x_{1}>250 \mathrm{mg} / \mathrm{dl}$ are denominated as level 2 hypoglycemia and level 2 hyperglycemia, respectively.

For all virtual patients evaluated, the performance statistics are computed for every strategy as recommended in [34], taking into account the fact that the results presented here are obtained from simulation and not from clinical trials. Table 2 includes the percentage of time in which glycemia is found both in and out of the normoglycemia zone and the amount of hypoglycemia and hyperglycemia events. Furthermore, the mean and standard deviation (SD) of BG with each controller are calculated. For each outcome the median and the interquartile range (IQR) of the population are shown, except for the mean and $\mathrm{SD}$ of $\mathrm{BG}$ concentration which are normally distributed so that the average and SD of the 11 patients are shown. The glycemia evolution for the 33 patients using the six different control strategies can be seen in the appendix, which shows that all controllers maintain the patients in the safe zone most of the time without risk of level 2 hypoglycemia. From the results, it is inferred that the iZMPC-AV presents a better performance than the other strategies. It has the highest percentage of precise control, achieving an $83.9 \%$ (IQR $11.8 \%$ ) of time inside the target zone with a median of 1 (IQR 2) event that exceeds $180 \mathrm{mg} / \mathrm{dl}$ corresponding to $1.3 \%$ (7.9\%) of the time. According to this, the iZMPC-AV is selected as the controller to be used in the offset-free control strategy, as was anticipated in early sections.

3.2. Sensitivity Analysis. The sensitivity function defined in (9) and given by the differential equation (10) is solved simultaneously with $x$ to obtain its time evolution. The proposed simulation scenario consists of two days, only one of which includes the intake of carbohydrates, with the purpose of demonstrating the sensitivity to parameters during fasting and when there is meal intake. The considered meals are $50 \mathrm{~g}$ at $7: 00 \mathrm{~h}, 80 \mathrm{~g}$ at $12: 00 \mathrm{~h}$, and $60 \mathrm{~g}$ at $21: 00 \mathrm{~h}$. In Figure 1, the magnitude of the sensitivity of glycemia to $p_{j}$, denoted as $\left\|S_{x_{1}}, p_{j}\right\|$, is plotted for the average adult as illustration. The parameters are categorized according to the behavior of the sensitivity magnitude, noticing similar responses to $p_{0}, p_{1}, p_{2}$, and $p_{3}$ associated with glycemia; $p_{4}, p_{5}, p_{6}$, and $p_{7}$ with insulin; and $p_{8}, p_{9}, p_{10}$, and $p_{11}$ with intake of carbohydrates. 

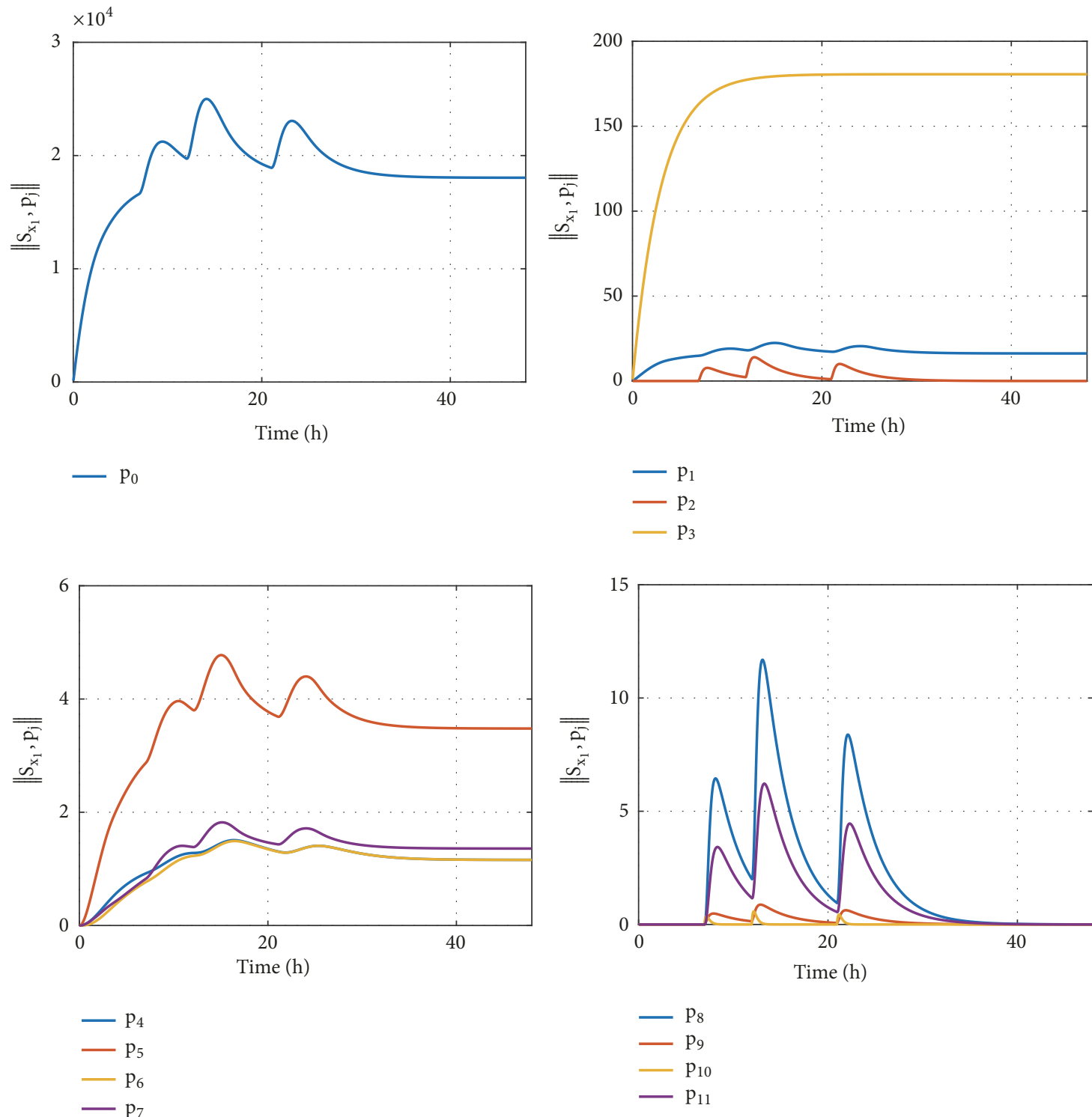

Figure 1: Glycemia sensitivity to each parameter of the model. Top: parameters associated with glycemia. Lower left: parameters associated with insulin. Lower right: parameters associated with intake of carbohydrates.

According to $\left\|S_{x_{1}}, p_{j}\right\|$, the parameters $p_{0}, p_{1}, p_{2}, p_{3}, p_{5}$, and $p_{8}$ are selected as the most influential in blood glucose concentration. Therefore, the simulation of the plant-model mismatch is done when variations in these parameters are considered. The closed-loop system with the iZMPC-AV control strategy is simulated for variations of $10 \%, 30 \%$, and $50 \%$ in the selected parameters and illustrated using the average patient. The behavior of glycemia in the second day confirms the offset provoked by these variations. In Figure 2, the effect of each of these is depicted and the following statements are established:

(i) By increasing the parameter $p_{0}$ in the plant, less amount of insulin is required. As the prediction model has a lower value of $p_{0}$, the controller overestimates the necessary dose of insulin and thus the glycemia decreases. If the value of $p_{0}$ is reduced, the required insulin is higher, but the controller underestimates the amount of insulin, which causes hyperglycemia.

(ii) Variations above the nominal value of $p_{1}$ steer to lower levels of BG, obtaining hypoglycemia events. This is because the patient is more sensitive to insulin and therefore requires a smaller amount in each dose, whereas, as the prediction model has a lower value of the parameter, the calculated insulin dose is higher than the actually needed. When insulin action effectiveness parameter is lower than the nominal value, the glycemia increases and hyperglycemia events may occur.

(iii) The variations in parameter $p_{2}$, corresponding to carbohydrate bioavailability, directly reduce or increase 

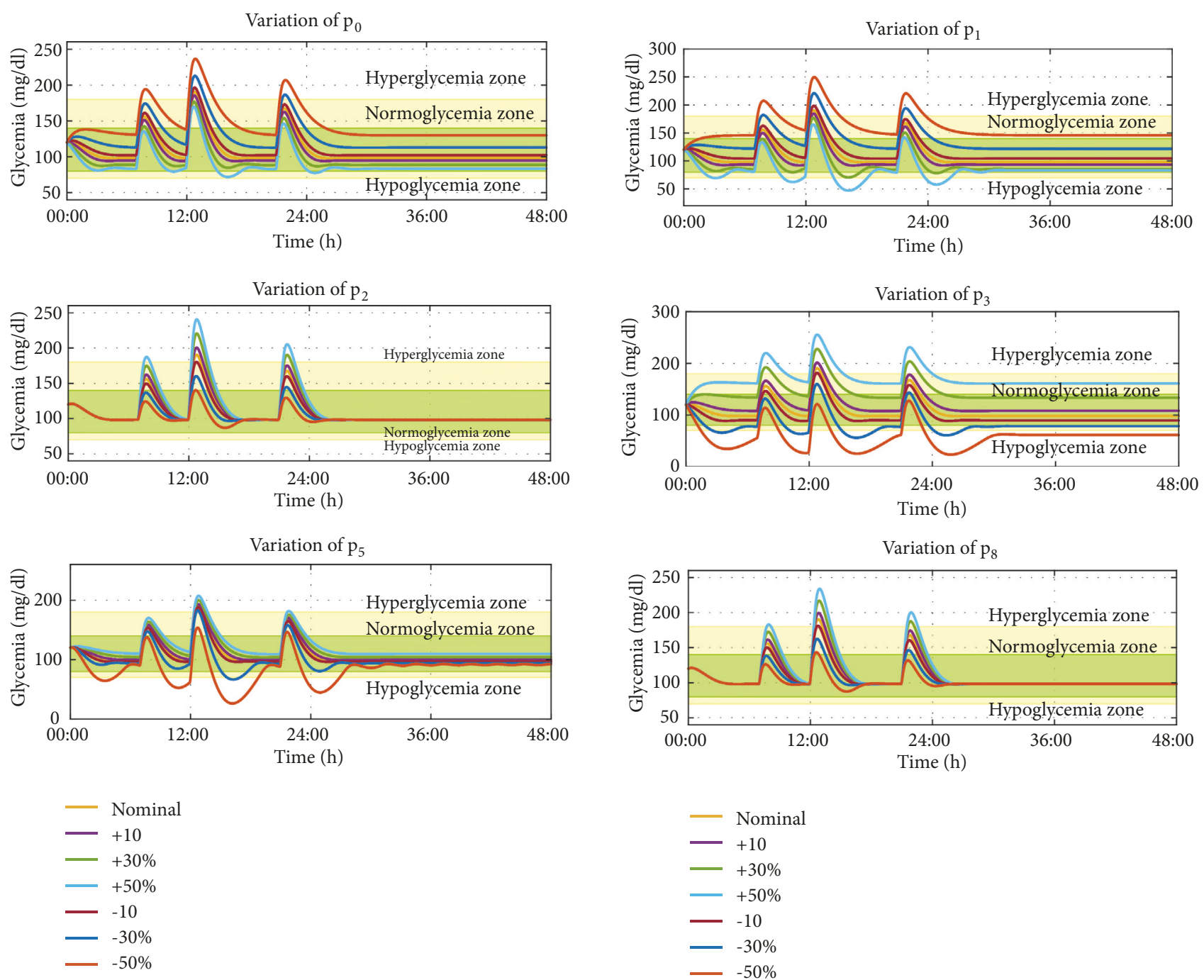

FIGURE 2: Variation of parameters $p_{0}, p_{1}, p_{2}, p_{3}, p_{5}$, and $p_{8}$ in $10 \%, 30 \%$, and $50 \%$ of their nominal values.

BG levels at the moment of the carbohydrate intake. When the real value of $p_{2}$ increases, the patient gets more sensitive to meal disturbances and episodes of hyperglycemia in the postprandial period are of greater magnitude. When the real value of $p_{2}$ is lower than the identified parameter, the effect of meals in the BG levels is reduced.

(iv) Variations in $p_{3}$ affect glycemia at the time of meals and at steady state. An increase in this parameter leads to an increment of glycemia and it tends to hyperglycemia events. A decrease in the parameter results in a reduction of BG levels and then in hypoglycemia events.

(v) Changes in the parameter $p_{5}$ are more critical at the time of meals and when its real value is less than the nominal value, leading to hypoglycemia episodes. When the effectiveness of insulin diffusion is greater, the glycemia rises. (vi) Variations in $p_{8}$ have an effect on glycemia only at the time of meals. When it is augmented, the glycemia is more sensitive to meal disturbances due to a longer time of diffusion of carbohydrates, leading the patient to have hyperglycemia episodes. By decreasing the value of the parameter, glycemia is reduced in the postprandial period. There is no offset presented because of its dependence on carbohydrate intake.

3.3. Offset-Free Strategy for Glycemia Control with PlantModel Mismatch. In this subsection, the iZMPC-AV strategy is affected by parameter variations. These variations normally appear in association with exercise, the dawn phenomenon, production of endogenous glucose, among others [35]. As was illustrated in the above subsection, depending on the magnitude of the parameter variation, the iZMPC-AV strategy cannot compensate the disturbance to maintain the adequate control. For that reason, the offset-free iZMPC$\mathrm{AV}$ (iZMPC-AV-OF) strategy is designed. Its performance is evaluated and compared with the iZMPC-AV without the 


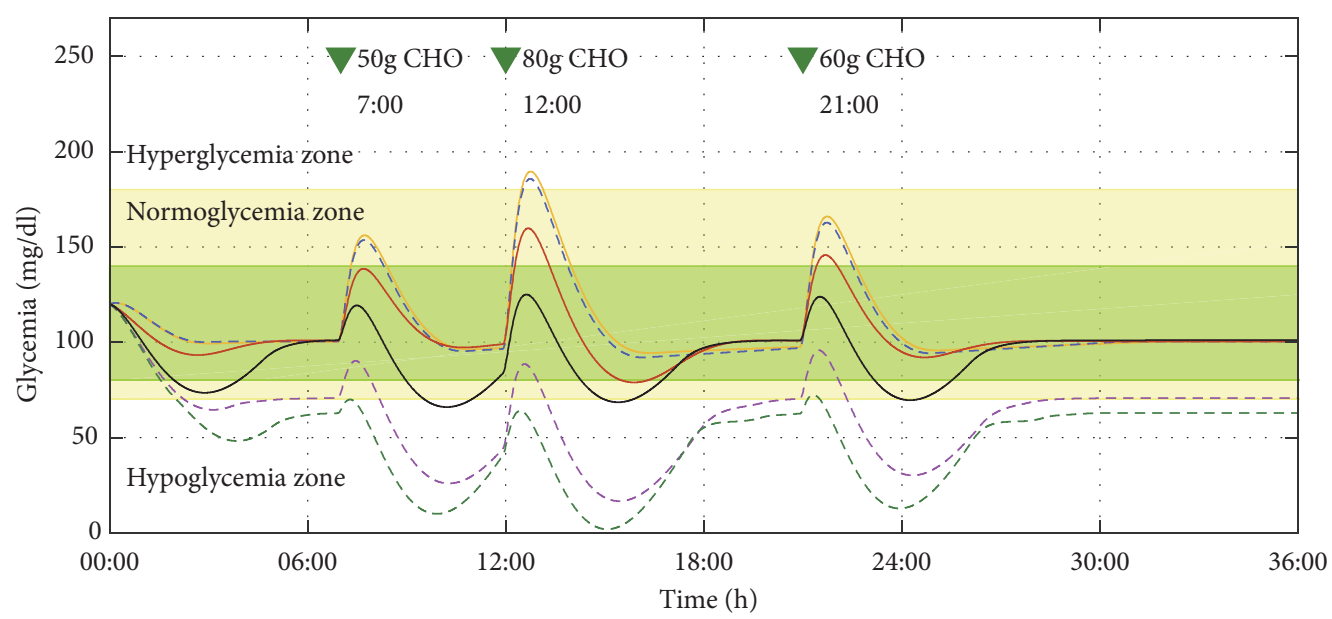

- - iZMPC-AV-NOF/Nominal model

- - iZMPC-AV-NOF/mismatch-30\%

- - iZMPC-AV-NOF/mismatch- $50 \%$

- iZMPC-AV-OF/Nominal model

- iZMPC-AV-OF/mismatch-30\%

- iZMPC-AV-OF/mismatch-50\%
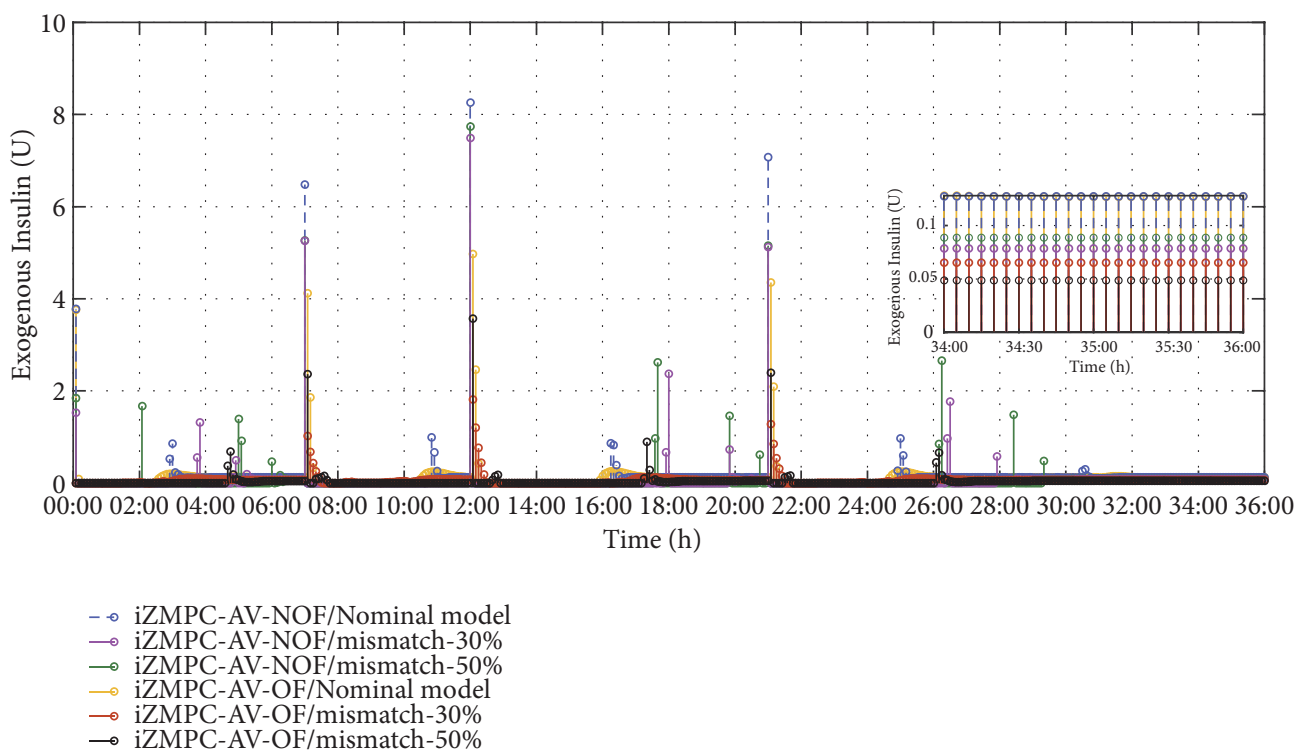

FIGURE 3: Comparison of glycemia evolution and insulin doses for the average adult patient under Scenario 2, considering variations of $0 \%, 30 \%$, and 50\%. Dashed lines (blue, purple, and green) represent iZMPC-AV-NOF while solid lines (yellow, red, and black) represent iZMPC-AV-OF.

offset-free strategy (iZMPC-AV-NOF) under the next two different scenarios:

Scenario 1. Variation of the parameters to induce hyperglycemia: the parameters $p_{2}, p_{3}, p_{5}$, and $p_{8}$ are increased with respect to their nominal values and parameters $p_{0}$ and $p_{1}$ are decreased by the same percentages.

Scenario 2. Variation of the parameters to induce hypoglycemia: the parameters $p_{0}$ and $p_{1}$ are increased with respect to their nominal values and parameters $p_{2}, p_{3}, p_{5}$, and $p_{8}$ are decreased by the same percentages.
As $x_{1}$ is the only measured state that corresponds to glycemia, the state variables $x_{2}, x_{3}, x_{4}$, and $x_{5}$ must be estimated, as well as the disturbance $d$ to correct the steady state mismatch. The simulation consists of 36 hours, starting at $0: 00 \mathrm{~h}$. The meals considered are a breakfast of $50 \mathrm{~g}$ at 7:00h, a lunch of $80 \mathrm{~g}$ at $12: 00 \mathrm{~h}$, and a dinner of $60 \mathrm{~g}$ at $21: 00 \mathrm{~h}$. The control objective is to drive glycemia to the zone between 70 $\mathrm{mg} / \mathrm{dl}$ and $180 \mathrm{mg} / \mathrm{dl}$ and remain there as long as possible. The control strategies do not consider meal announcements.

To illustrate the resulting effect when the offset-free strategy is used, Figure 3 depicts a comparison of the evolution of the glycemia and the insulin doses for the average 


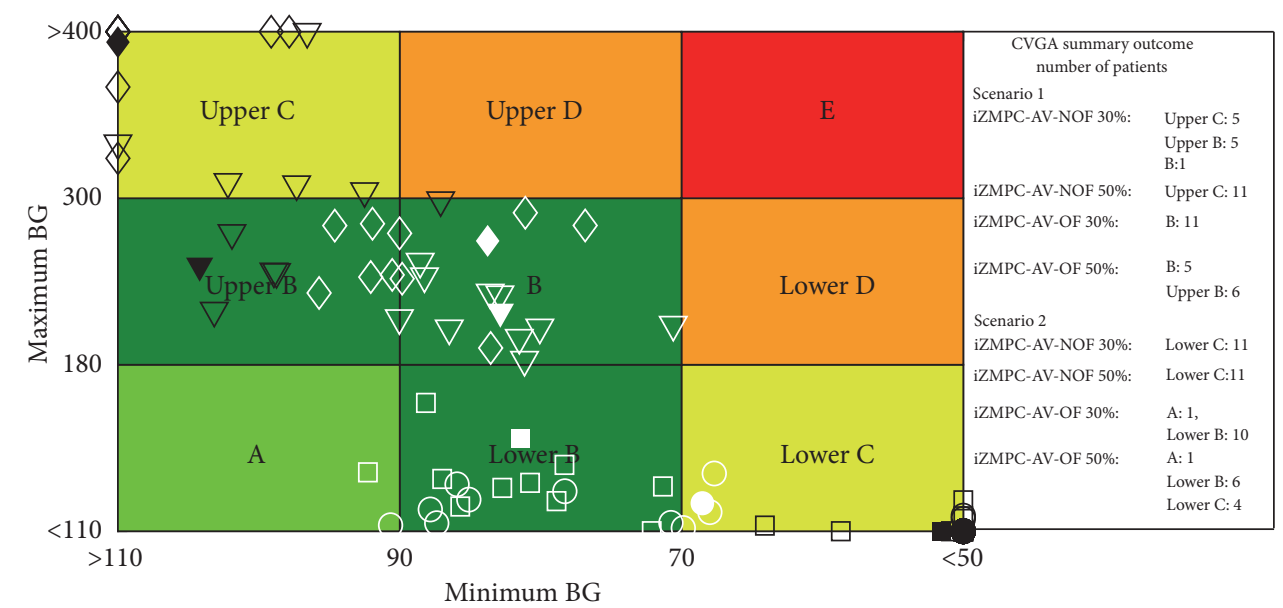

FIGURE 4: CVGA comparison of the iZMPC-AV-NOF (black) and iZMPC-AV-OF (white) strategies for 11 adult patients for each variation scenario. Scenario 1: the triangle mark is used for the $30 \%$ variation, and the diamond for $50 \%$ variation. Scenario 2: the square mark is used for the $30 \%$ variation, and the circle for $50 \%$ variation. The average patient in all cases is represented for the filled form.

adult patient under Scenario 2 considering variations of $0 \%$ (nominal situation), 30\%, and 50\%. The dashed lines are produced when the plant is controlled with the iZMPC-AVNOF strategy, and the solid lines when the iZMPC-AV-OF strategy is applied. The maximum variation in parameters before the occurrence of hypoglycemia events (or prolonged hyperglycemia events) is established at $50 \%$ of the nominal values of the parameters. Meals are only given on the first day to expose the correction at steady state of the second day.

It is noticed that with the iZMPC-AV-NOF the glycemia levels are adequately regulated when the plant and the model are equal, but they decrease to critical BG levels when there are parameter variations of $30 \%$ and $50 \%$, maintaining glycemia below $50 \mathrm{mg} / \mathrm{dl}$ at meal times. At steady state (from $30: 00 \mathrm{~h}$ to $36: 00 \mathrm{~h}$ ), the offset is well appreciated since the glycemia is out of the target zone. This behavior shows the need to apply a strategy that compensates the plant-model mismatch. On the other hand, the iZMPC-AV-OF manages to maintain the glycemia in the desired zone despite the variations. For variations of $30 \%$, the glycemia remains above $80 \mathrm{mg} / \mathrm{dl}$, and for variations of $50 \%$ the glycemia remains between 70 and $180 \mathrm{mg} / \mathrm{dl}$ and the effect of meal disturbances is reduced. When the iZMPC-AV-OF is applied to the nominal model, the behavior is very similar to that obtained with the iZMPC-AV-NOF; there are negligible differences at meal times, but at steady state the behavior is the same.

Regarding the control action for both strategies, when there is a plant-model mismatch and the iZMPC-AV-NOF is applied, it is observed that the offset at steady state is given by a miscalculation of the doses of insulin that are administered. The basal insulin is greater than that needed for the plant with the mismatch. In contrast, when applying the iZMPC$\mathrm{AV}-\mathrm{OF}$ strategy, the amount of basal insulin administered is corrected, and the dose is reduced. Finally, for the nominal model, it is seen that the basal has the same magnitude for the iZMPC-AV-NOF and the iZMPC-AV-OF.

The performances of iZMPC-AV-NOF and iZMPC-AVOF, both in Scenarios 1 and 2, are compared using the Control
Variability Grid Analysis (CVGA) [36]. Figure 4 shows the CVGA for the 10 adult patients and the average adult patient. Black color corresponds to the extreme glucose excursions for the 11 patients when the iZMPC-AV-NOF is used, and white color corresponds to the extreme excursions when the iZMPC-AV-OF is used. For Scenario 1, variations of $30 \%$ and $50 \%$ are represented by the triangle and diamond marker, respectively. For Scenario 2, 30\%, and 50\% variations are represented by the square and the circle marker, respectively.

It is evident that the iZMPC-AV-OF achieves better results than the iZMPC-AV-NOF for each case. For Scenario 1 , it can be seen that with the iZMPC-AV-NOF most patients have excursions in level 2 hyperglycemia, obtaining excursions greater than $400 \mathrm{mg} / \mathrm{dl}$ for variations of $50 \%$. Meanwhile, with the iZMPC-AV-OF strategy, the population is moved to lower levels of BG; for variations of $30 \%$, the iZMPC-AV-OF maintains all patients with benign control deviations (zone B), and for 50\% variations, 55\% of the cases show benign deviations into hyperglycemia (zone upper B). In Scenario 2, more critical results are obtained with the iZMPC-AV-NOF strategy, since there are only 4 cases for variations of $30 \%$ that do not present level 2 hypoglycemia. When applying the iZMPC-AV-OF strategy, the BG levels are augmented, moving the population to a safe control (zone $A+B)$, and for variations of $50 \%$ there is a safe control in $64 \%$ of the cases and an overcorrection of hyperglycemia (zone lower C) for $36 \%$ of the cases.

Figure 5 shows the median of the 11 patients when applying the iZMPC-AV-OF strategy for the same simulation scenarios described above. In addition, the region between the $25^{\text {th }}$ and $75^{\text {th }}$ percentiles of the glycemia is displayed. From this range, it can be observed that in all cases the BG is regulated within the target zone at steady state. The minimum glycemia curves show that there are no level 2 hypoglycemia events. For variations to induce hypoglycemia (Scenario 2), the $\mathrm{BG}$ is maintained in the normoglycemia zone for all patients, and then the risk of postprandial hyperglycemia and induced hypoglycemia is eliminated. 


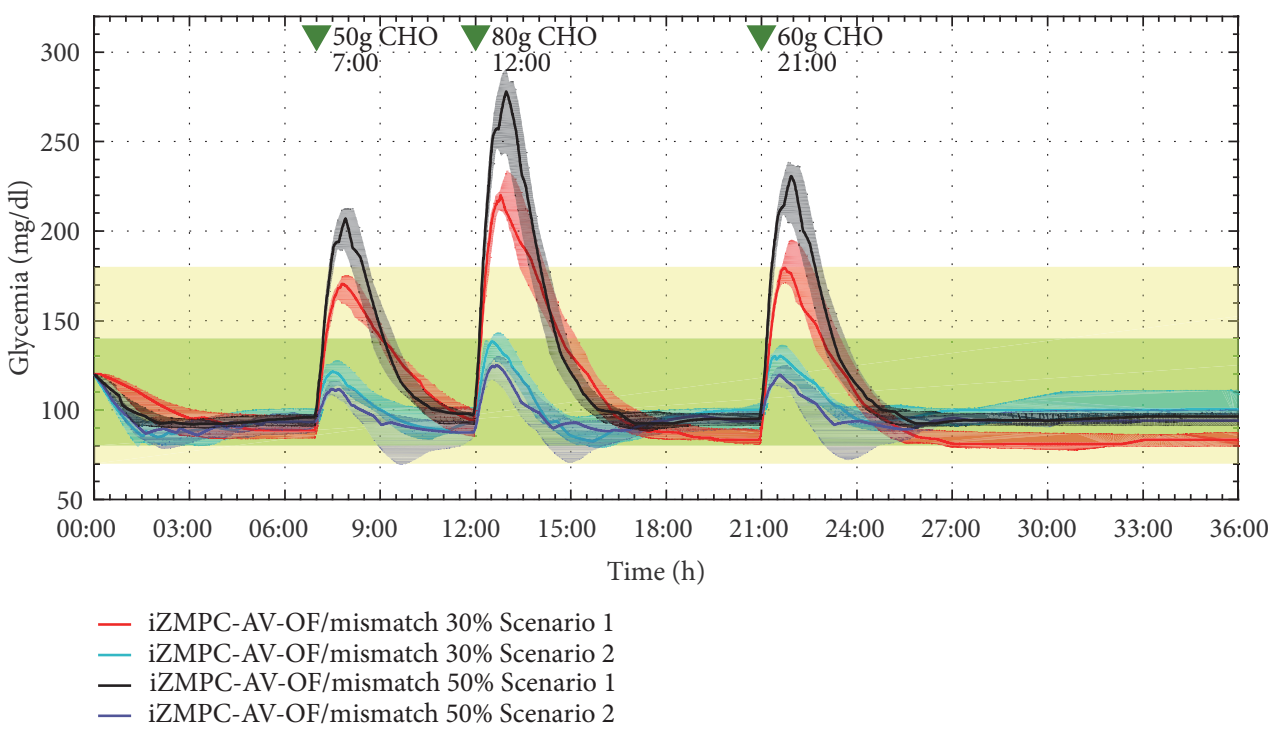

Figure 5: Glycemia evolution of adult patients when variations in the parameters of $30 \%$ and 50\% in Scenario 1 (to induce hyperglycemia) and Scenario 2 (to induce hypoglycemia) are presented and the iZMPC-AV-OF strategy is used. Continuous lines represent the $50^{\text {th }}$ percentile of the glycemia (median), and the shaded regions are the $25^{\text {th }}$ to $75^{\text {th }}$ percentiles of each case.

The glucose control metrics of the 11 patients in each variation scenario to induce hyperglycemia and hypoglycemia are shown in Table 3 . From the results with the iZMPC$\mathrm{AV}-\mathrm{NOF}$ it is possible to reaffirm the need for an extra strategy to compensate the plant-model mismatch. With each variation, the percentage of time in normoglycemia decreases significantly, obtaining 30.0\% (IQR 3.3\%) of the time in normoglycemia with the variations of $50 \%$ in Scenario 1 and $38.2 \%(45.3 \%)$ in Scenario 2, and driving BG to level 2 hyperglycemia and level 2 hypoglycemia during a high percentage of time, $43.4 \%(10.2 \%)$ and $37.5 \%(35.4 \%)$, respectively. On the other hand, a greater percentage of time can be noticed in the target when the iZMPC-AV-OF is applied. In Scenario 1, for variations of the parameters to induce hyperglycemia, it is possible to maintain the glycemia in the zone $70-140 \mathrm{mg} / \mathrm{dl}$ for a percentage of time greater than $70 \%$. Only $3.6 \%(4.2 \%)$ of the time is obtained in level 2 hyperglycemia for variations up to $50 \%$, reaching a maximum of $306.04 \mathrm{mg} / \mathrm{dl}$ in a patient. In Scenario 2, when variations to induce hypoglycemia are made, a significant increase in the percentage of time in the same zone is noticed, maintaining glycemia in the target more than $90 \%$ of the time. In this scenario there are no episodes of level 2 hyperglycemia or hypoglycemia. For greater variations of the parameters in Scenario 1 , the percentage of time in the target zone is less than $70 \%$, so that it is considered that variations up to $50 \%$ are adequately compensated with the iZMPC-AV-OF strategy.

To complement the results obtained with the offset-free strategy, a comparison between the dZMPC without offsetfree strategy (dZMPC-NOF) and the offset-free dZMPC (dZMPC-OF) is done in order to illustrate the effect in a discrete scheme. For that goal, in Figure 6 the same simulation scenarios used in Figure 3 are taken into account. Then it shows a comparison of the evolution of the glycemia and the insulin doses for the average adult patient.
As observed with the iZMPC-AV-NOF, the dZMPC-NOF adequately regulates the glycemia when there is no plantmodel mismatch, but BG levels fall below $54 \mathrm{mg} / \mathrm{dl}$ when there are parameter variations of $30 \%$ and $50 \%$. Contrarily, with the dZMPC-OF, the glycemia remains in the desired zone despite the variations; it completely eliminates the offset at steady state and reduces the effect of meal intake. However, it is observed that for $30 \%$ variations, BG levels fall almost to the limit of normoglycemia due to the late postprandial effect, which was not evidenced with the iZMPC-AV-OF in Figure 3 for the same percentage of variation. Furthermore, it can be seen that the offset in steady state with the dZMPC-NOF is given by a miscalculation of insulin doses, administering a greater amount of basal insulin than that needed for the patient; when applying the offset-free strategy, the amount of basal insulin administered is reduced. Finally, from Figures 3 and 6 , the difference of the control action of a discrete system and an impulsive system can be recognized. In the impulsive case, the magnitude of each infusion of insulin is greater than that in the discrete case, because insulin is not delivered continuously but in every period of time.

The performance metrics obtained with the dZMPCNOF and dZMPC-OF are reported in Table 4. The improvement obtained by applying the dZMPC-OF in relation to the dZMPC-NOF is evident. For the variations of $50 \%$, in Scenario $1,8.5 \%$ (IQR $32.9 \%$ ) of the time in normoglycemia results when the dZMPC-NOF is used compared to $71.1 \%$ $(13.0 \%)$ of the time when the dZMPC-OF is used; there is also a big difference in Scenario 2, since there is only $29.7 \%$ (15.6\%) of time in normoglycemia with the dZMPC-NOF compared to $95.8 \%(10.3 \%)$ with the dZMPC-OF. It is possible to see how the mean of BG is reduced in Scenario 1 and increased in Scenario 2, reaching the desired zone, when applying the dZMPC-OF for each variation. 


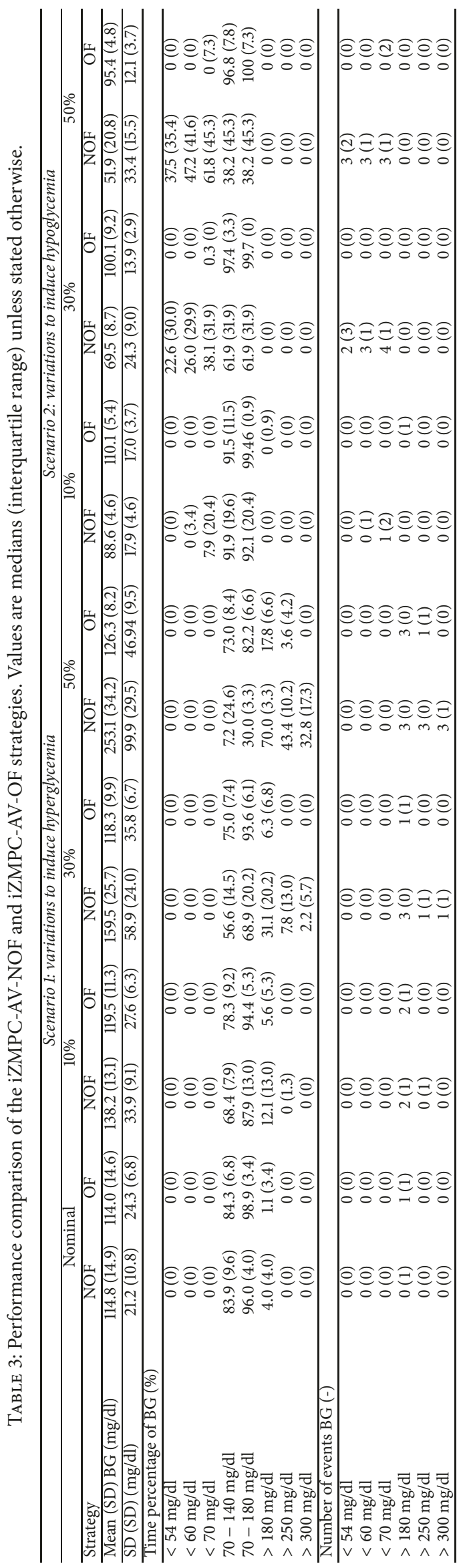




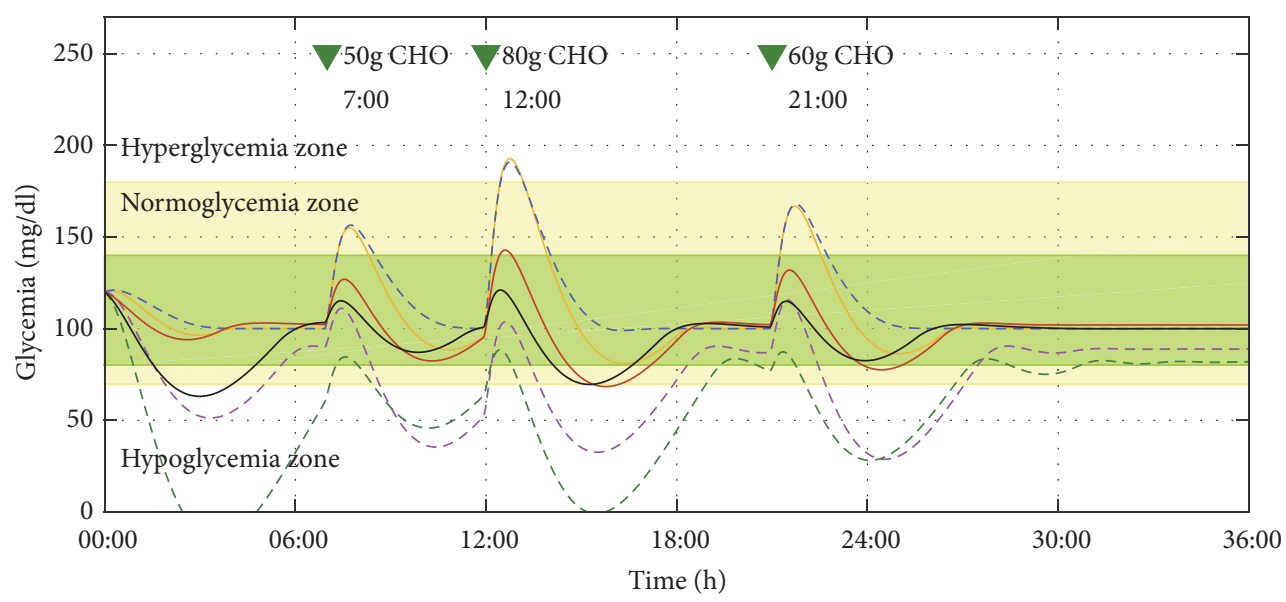

- - dZMPC-NOF/Nominal model

- - dZMPC-NOF/mismatch-30\%

- - dZMPC-NOF/mismatch-50\%

- dZMPC-OF/Nominal model

- dZMPC-OF/mismatch-30\%

- dZMPC-OF/mismatch-50\%

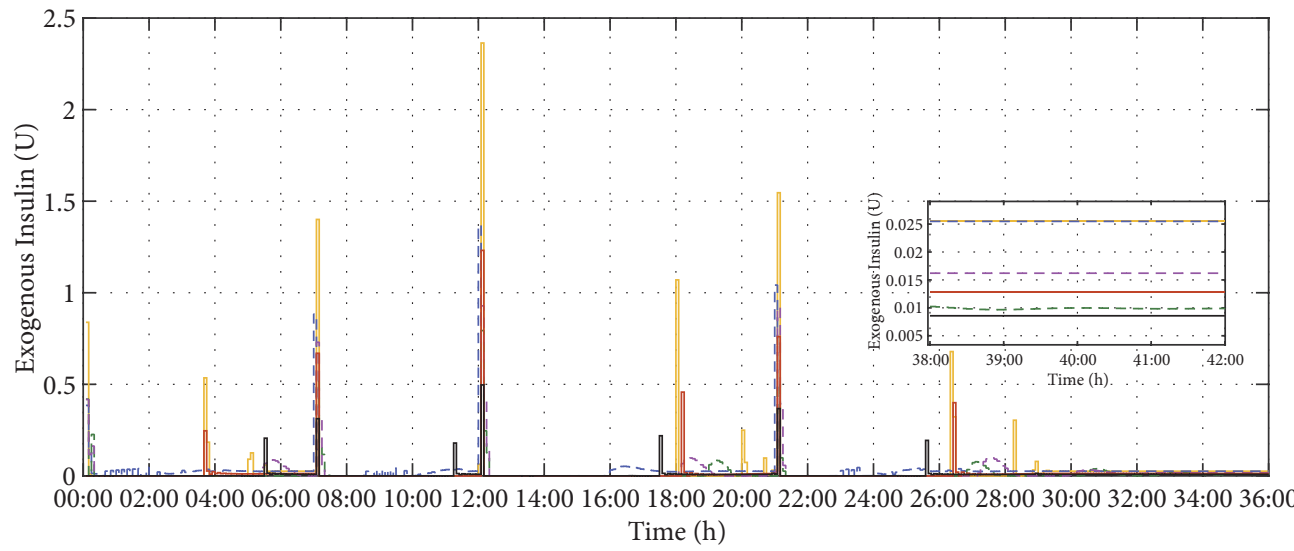

- dZMPC/Nominal model

_. $\mathrm{dZMPC} / \mathrm{mismatch}-30 \%$

_. $\mathrm{dZMPC/mismatch-50 \%}$

dZMPC-OF/Nominal model

- dZMPC-OF/Nominal model

- dZMPC-OF/mismatch-50\%

FIGURE 6: Comparison of glycemia evolution and insulin doses for the average adult patient under Scenario 2, considering variations of $0 \%, 30 \%$, and 50\%. Dashed lines (blue, purple, and green) are used for dZMPC-NOF while solid lines (yellow, red, and black) are used for dZMPC-OF.

Lastly, in Figure 7 the median of the 11 patients and the region between the $25^{\text {th }}$ and $75^{\text {th }}$ percentiles of the glycemia is displayed when applying the iZMPC-AV-OF strategy considering a robustness test with different meals, parameter variations of $30 \%$ in Scenario 2, and sensor noise (i.e., the CGM signal is used instead of the glycemia). The meals consist of $50 \mathrm{~g}$ at $6: 00 \mathrm{~h}, 20 \mathrm{~g}$ at $9: 00 \mathrm{~h}, 90 \mathrm{~g}$ at $13: 00 \mathrm{~h}$, $20 \mathrm{~g}$ at $17: 00 \mathrm{~h}$, and $70 \mathrm{~g}$ at 22:00h. The accuracy and dynamics of continuous glucose sensors have been studied in [37]. The noise is then modeled as an ARMA process with a non-Gaussian noise following the Johnson distribution. The percentage of time in the target zone corresponds to $81.56 \%$; it has 15 events of level 1 hypoglycemia and 2 events of level
2 hypoglycemia corresponding to $6.82 \%$ and $0.25 \%$ of the time, respectively; there is one event of hyperglycemia with a percentage of time of $2.84 \%$. It is noticed that, during the second day, the glycemia reaches its steady state without offset (it achieves the target zone).

\section{Conclusions}

A performance comparison of the different MPC strategies for discrete and impulsive systems, applied to the T1DM treatment problem, was done in a simulation environment by means of the UVa/Padova simulator. All these strategies were tested against meal disturbances, without announcement to 


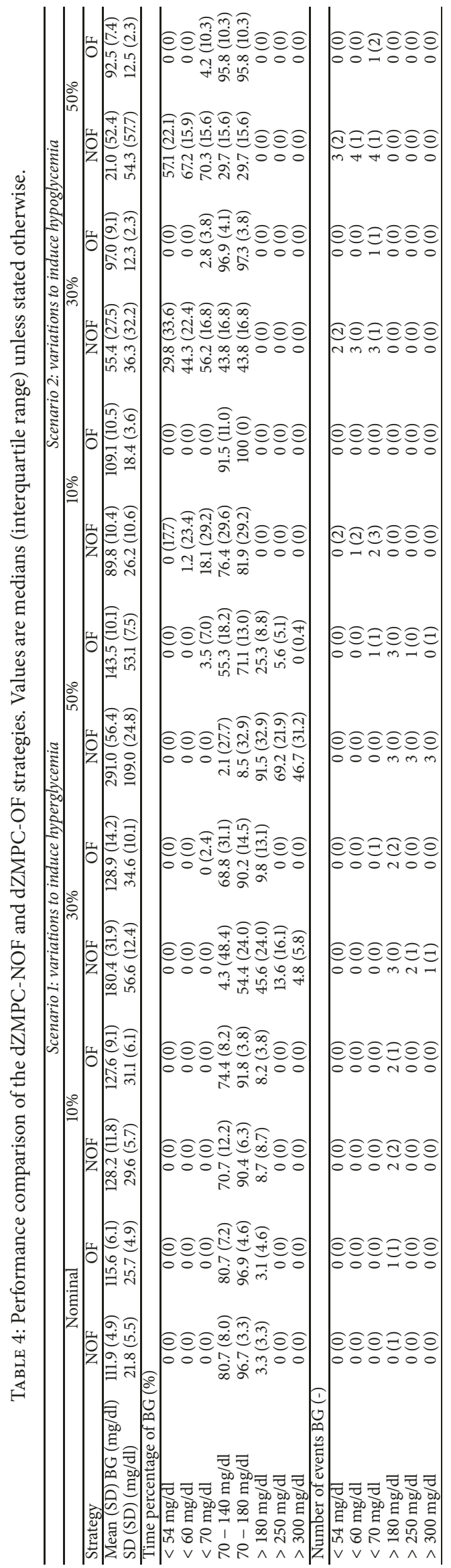




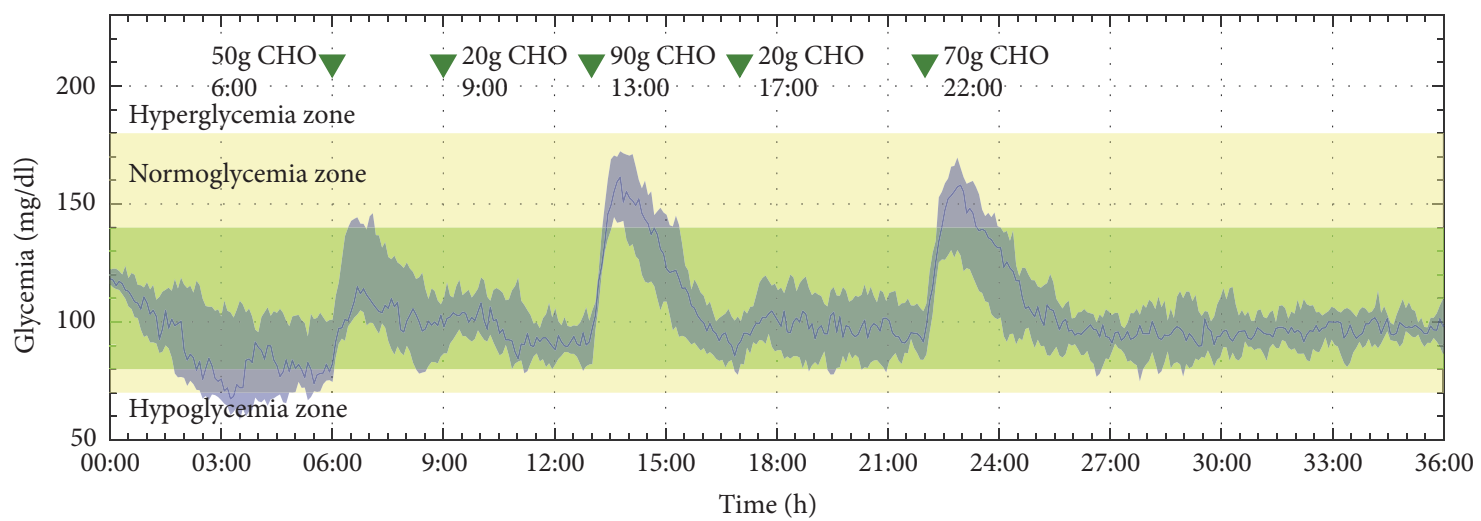

FIGURE 7: Glycemia evolution of adult patients when the iZMPC-AV-OF strategy is applied. There are variations of 30\% in Scenario 2, sensor noise is considered, and five meals are provided. Continuous lines represent the median, and the shaded regions are the $25^{\text {th }}$ to $75^{\text {th }}$ percentiles of each case.

the control strategy. The use of ZMPC strategies seems to be adequate for the T1DM treatment, since it applies enough insulin to maintain the blood glucose in the target zone, eliminating the need to administer greater amount of insulin in each dose to reach a set-point and fulfil constraints. The iZMPC-AV presents the least percentage of time out of the normoglycemia zone. One of its main benefits is that it has an enlarged domain of attraction due to the artificial intermediary variables and the application of the basal as small boluses instead of a continuous dose because of the impulsive scheme.

The main goal of this work is to propose a control strategy based on the iZMPC-AV for the glycemic regulation problem in type 1 diabetic patients, including a plant-model mismatch caused by variations in the parameters. The iZMPC-AVOF strategy is then used to treat the problem. The main achievement of this control application is to maintain the blood glucose levels within the target zone of normoglycemia, decreasing hypoglycemia and hyperglycemia events caused by parameter variations and carbohydrate intake.

By simulating two scenarios for 11 adult virtual patients, satisfactory performances were achieved. It was shown that the dZMPC-OF and iZMPC-AV-OF strategies maintain the glycemia in the normal range $(70-180 \mathrm{mg} / \mathrm{dl})$ for variations up to $50 \%$. Hypoglycemia episodes were successfully avoided and hyperglycemia events were minimized. In Scenario 1, when the variations are made to induce hyperglycemia, the percentage time in the target exceeds $70 \%$ by applying the iZMPC-AV-OF strategy, while in Scenario 2, when variations to induce hypoglycemia are made, the percentage of time increases over $90 \%$. This is because of two main reasons: (i) variations in the parameters, in Scenario 2, associated with carbohydrates favor the reduction of hyperglycemia peaks in postprandial times, and (ii) the controller manages to reduce the episodes of hypoglycemia, maintaining glycemia in the target zone for approximately $90 \%$ of the time.

This work presents a first step to counteract the plantmodel mismatch inherent in a biomedical problem such as the treatment of type 1 diabetes mellitus. The results are obtained in a limited simulation environment built with the
UVa/Padova simulator, which tend to have more contraregulation on low glycemic values than that observed in real subjects. Although the results are very good, it is necessary to remember that the simulated scenarios do not exactly describe real-life conditions. For further works more realistic scenarios will be taken into account, including temporary disturbances in the parameters given by exercise, the dawn phenomenon, insulin sensitivity, and the time constant of insulin absorption. In addition, an analysis of the noise given by the glucose sensor must be considered in more detail, which, having a nonzero-mean, can alter the elimination of the offset.

\section{Appendix}

\section{A. MPC Formulations to Treat Type 1 Diabetes}

A.1. Discrete-Time Formulations. The discrete control strategies are formulated taking into account the discrete-time affine model of the form

$$
\begin{aligned}
x(k+1) & =A_{D} x(k)+B_{u D} u(k)+B_{r D} r(k)+E_{D}, \\
x(0)=x_{0}, & \\
y(k) & =C_{D} x(k),
\end{aligned}
$$

where matrices $A_{D}, B_{u D}, B_{r D}, E_{D}$, and $C_{D}$ are the discrete matrices of system 2.1 .

A.1.1. Standard Discrete MPC (dMPC) [24]. The cost function to be minimized is quadratic; it penalizes the deviation of the state $x$ with respect to a reference $x_{r e f}$, the deviation of the input $u$, and the reference input $u_{\text {ref }}$ to achieve the state target and a terminal cost to ensure convergence at the end of the prediction horizon $N$ :

$$
\begin{aligned}
& V_{N}\left(x, r, x_{r e f}, u_{r e f} ; \boldsymbol{u}\right) \\
& \quad=V_{d y n}\left(x, r, x_{r e f}, u_{r e f} ; \boldsymbol{u}\right)+V_{f}\left(x, r, x_{r e f}\right),
\end{aligned}
$$




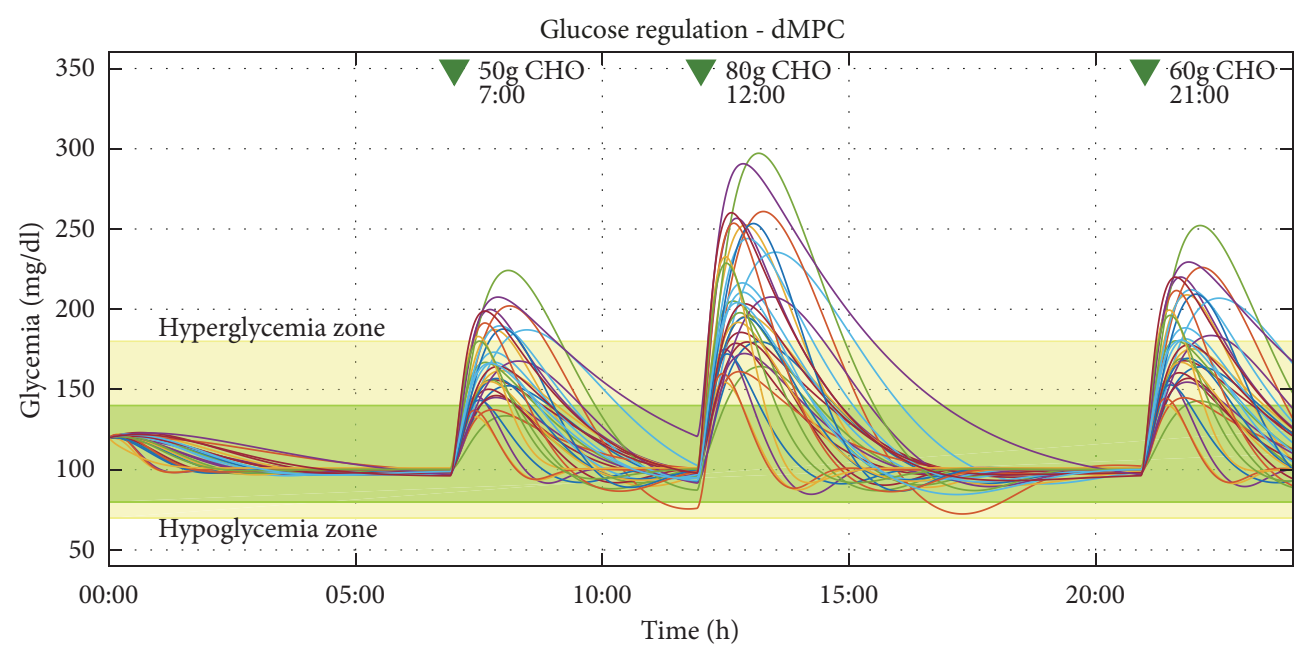

Target zone

Normoglycemia zone

FIGURE 8: Blood glucose regulation for 33 virtual patients using the dMPC.

where $V_{d y n}$ and $V_{f}$ are

$$
\begin{aligned}
& V_{d y n}\left(x, r, x_{r e f}, u_{r e f} ; \boldsymbol{u}\right)=\sum_{i=0}^{N-1}\left\|x(k+i \mid k)-x_{r e f}\right\|_{Q}^{2} \\
& \quad+\left\|u(k+i \mid k)-u_{r e f}\right\|_{R}^{2}, \\
& V_{f}\left(x, r, x_{r e f}\right)=\left\|x(k+N \mid k)-x_{r e f}\right\|_{P}^{2} .
\end{aligned}
$$

Considering the state and input constraints, the MPC problem to be solved at each time step $k$ is

$$
\begin{array}{ll}
\min _{\boldsymbol{u}} & V_{N}\left(x, r, x_{r e f}, u_{r e f} ; \boldsymbol{u}\right) \\
\text { s.t. } & x(0)=x(k \mid k), \\
& x(k+i \mid k) \\
& =A_{D} x(k+i-1 \mid k)+B_{u D} u(k+i-1 \mid k) \\
& +B_{r D} r(k+i-1 \mid k)+E_{D}, \\
& u(k+i \mid k) \in U, \\
& x(k+i \mid k) \in X,
\end{array}
$$

in which the solution is the input trajectory $\boldsymbol{u}=\{u(k \mid$ $k), \ldots, u(k+N-1 \mid k)\}$, and the first element of the sequence $u(k \mid k)$ is applied to the plant. Figure 8 depicts the glycemia time evolution for the 33 patients of the UVa/Padova simulator when the dMPC strategy is applied to the system. The scenario of simulation is explained in Section 3.1.

A.1.2. Discrete Zone MPC (dZMPC) [26]. The dZMPC is formulated adding a new decision variable $\delta$ and defining its upper and lower limits; this creates a zone where the cost is zero when the predicted variables are inside the target zone and different from zero when they are outside it. The cost function is then given by

$$
\begin{aligned}
& V_{N}\left(x, r, x_{r e f}, u_{r e f} ; \boldsymbol{u}, \delta\right) \\
& \quad=V_{d y n}\left(x, r, x_{r e f}, u_{r e f} ; \boldsymbol{u}, \delta\right)+V_{f}\left(x, r, x_{r e f} ; \delta\right),
\end{aligned}
$$

where $V_{d y n}$ and $V_{f}$ are

$$
\begin{aligned}
& V_{d y n}\left(x, r, x_{r e f}, u_{r e f} ; \boldsymbol{u}, \delta\right) \\
& =\sum_{i=0}^{N-1}\left\|x(k+i \mid k)-x_{r e f}+\delta\right\|_{Q}^{2} \\
& \quad+\left\|u(k+i \mid k)-u_{r e f}\right\|_{R}^{2}, \\
& V_{f}\left(x, r, x_{r e f} ; \delta\right)=\left\|x(k+N \mid k)-x_{r e f}+\delta\right\|_{P}^{2} .
\end{aligned}
$$

The parameters $x_{r e f}$ and $u_{r e f}$ are selected as a reference inside the target zone. Considering the state and input constraints, the MPC problem to be solved at each time step $k$ is

$$
\begin{array}{ll}
\min _{\boldsymbol{u}, \delta} & V_{N}\left(x, r, x_{r e f}, u_{r e f} ; \boldsymbol{u}, \delta\right) \\
\text { s.t. } & x(0)=x(k \mid k), \\
& x(k+i \mid k) \\
& =A_{D} x(k+i-1 \mid k)+B_{u D} u(k+i-1 \mid k) \\
& \quad+B_{r D} r(k+i-1 \mid k)+E_{D}, \\
& u(k+i \mid k) \in U, \\
& x(k+i \mid k) \in X, \\
& \delta_{\text {min }} \leq \delta \leq \delta_{\text {max }},
\end{array}
$$




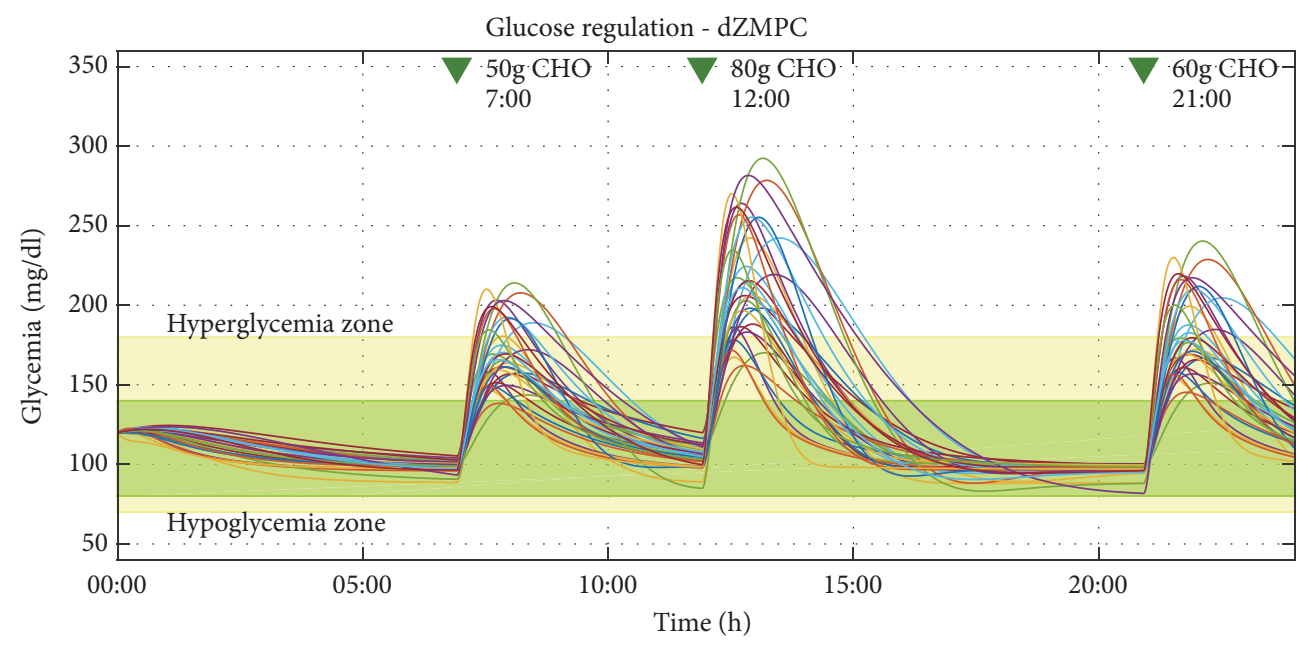

Target zone

Normoglycemia zone

FIGURE 9: Blood glucose regulation for 33 virtual patients using dZMPC.

in which the solution is the input trajectory $\boldsymbol{u}=\{u(k \mid$ $k), \ldots, u(k+N-1 \mid k)\}$, and the first element of the sequence $u(k \mid k)$ is applied to the plant. Figure 9 shows results of this strategy under the scenario defined in Section 3.1.

A.1.3. Discrete Zone MPC with Artificial Variables (dZMPC$A V$ ) [29]. In this formulation the set-point concept is changed and the system is lead to a target equilibrium set $X_{s}^{\text {Tar }} \subseteq X_{s}$, with its associated input set $U_{s}^{\text {Tar }} \subseteq U_{s}$, where $X_{s}$ and $U_{s}$ are the equilibrium sets of the system. The iZMPC-AV consist of four ingredients: (i) the use of artificial/intermediary equilibrium variables $\left(x_{a}, u_{a}\right) \in X_{s} \times$ $U_{s}$, (ii) a cost to penalize the deviation between the predicted state and input $(x, u)$ and the artificial equilibrium $\left(x_{a}, u_{a}\right)$, (iii) a terminal cost to penalize the deviation between the artificial variables and the target sets $X_{s}^{T a r}, U_{s}^{T a r}$, and (iv) a terminal constraint to guarantee that the state $x$ achieves the artificial variable at the end of the horizon. The cost function is

$$
\begin{aligned}
& V_{N}\left(x, r, X_{s}^{\mathrm{Tar}}, U_{s}^{\mathrm{Tar}} ; \boldsymbol{u}, u_{a}, x_{a}\right) \\
& =V_{d y n}\left(x, r ; \boldsymbol{u}, x_{a}, u_{a}\right) \\
& \quad+V_{f}\left(X_{s}^{\mathrm{Tar}}, U_{s}^{\mathrm{Tar}} ; x_{a}, u_{a}\right),
\end{aligned}
$$

where $V_{d y n}$ and $V_{f}$ are as follows.

$$
\begin{aligned}
& V_{d y n}\left(x, r ; \boldsymbol{u}, x_{a}, u_{a}\right) \\
& =\sum_{i=0}^{N-1}\left\|x(k+i \mid k)-x_{a}\right\|_{\mathrm{Q}}^{2} \\
& \quad+\sum_{i=0}^{N-1}\left\|u(k+i \mid k)-u_{a}\right\|_{R}^{2},
\end{aligned}
$$

$$
\begin{aligned}
V_{f} & \left(X_{s}^{\text {Tar }}, U_{s}^{\text {Tar }} ; x_{a}, u_{a}\right) \\
& =P\left(\operatorname{dist}_{X_{s}^{\text {Tar }}}\left(x_{a}\right)+\operatorname{dist}_{U_{s}^{\text {Tar }}}\left(u_{a}\right)\right)
\end{aligned}
$$

The MPC problem is

$$
\begin{array}{ll}
\min _{\boldsymbol{u}, u_{a}, x_{a}} & V_{N}\left(x, r, X_{s}^{T a r}, U_{s}^{T a r} ; \boldsymbol{u}, u_{a}, x_{a}\right) \\
\text { s.t. } \quad & x(0)=x(k \mid k), \\
& x(k+i \mid k) \\
= & A_{D} x(k+i-1 \mid k) \\
& +B_{u D} u(k+i-1 \mid k) \\
& +B_{r D} r(k+i-1 \mid k)+E_{D}, \\
& u(k+i \mid k) \in U, \\
x & x+i \mid k) \in X, \\
x & x+N \mid k)=x_{a}, \\
x & x_{a}=A_{D} x_{a}+B_{D} u_{a}+E_{D},
\end{array}
$$

in which the solution is the input trajectory $\boldsymbol{u}=\{u(k \mid$ $k), \ldots, u(k+N-1 \mid k)\}$, and the first element of the sequence $u(k \mid k)$ is applied to the plant. Note that the artificial variables are now decision variables. Figure 10 shows the results of this strategy under the scenario defined in Section 3.1.

A.2. Impulsive-Time Formulations. For the next three MPC formulations, the considered model is the underlying discrete-time subsystem (3) that represents the impulsive system (2) at the impulse times $\tau_{k}$. 


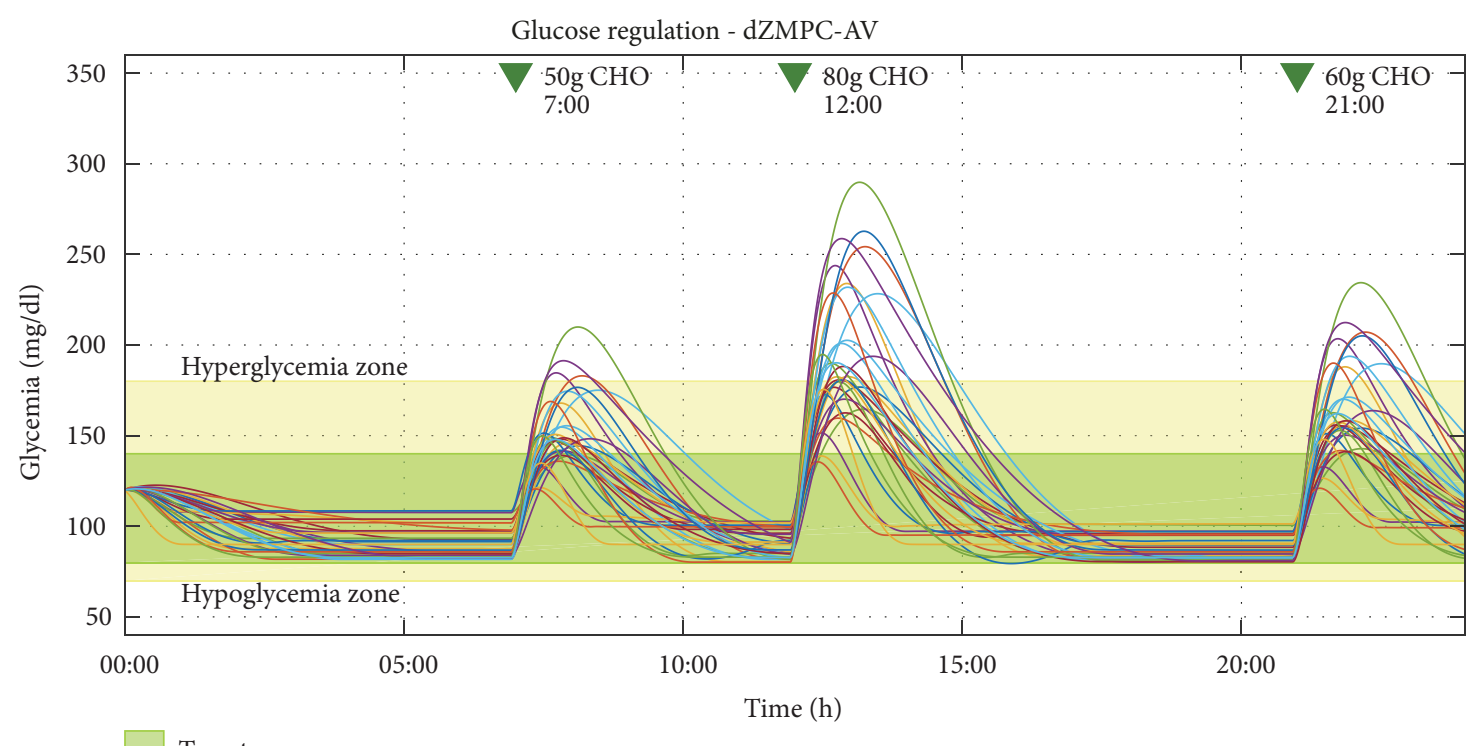

Target zone

Normoglycemia zone

FIGURE 10: Blood glucose regulation for 33 virtual patients using the dZMPC-AV.

A.2.1. Standard Impulsive MPC (iMPC) [30]. The cost function to be minimized has the same form as ((A.2)-(A.4)) to penalize the deviation of the state and input $(x, u)$ with respect to a reference $\left(x_{r e f}, u_{r e f}\right)$

$$
\begin{aligned}
& V_{N}\left(x^{\bullet}, r^{\bullet}, x_{r e f}, u_{r e f} ; \boldsymbol{u}\right) \\
& \quad=V_{d y n}\left(x^{\bullet}, r^{\bullet}, x_{r e f}, u_{r e f} ; \boldsymbol{u}\right)+V_{f}\left(x^{\bullet}, r^{\bullet}, x_{r e f}\right),
\end{aligned}
$$

where $V_{d y n}$ and $V_{f}$ are

$$
\begin{aligned}
& V_{d y n}\left(x^{\bullet}, r^{\bullet}, x_{r e f}, u_{r e f} ; \boldsymbol{u}\right) \\
& \quad=\sum_{i=0}^{N-1}\left\|x^{\bullet}(k+i \mid k)-x_{r e f}\right\|_{Q}^{2} \\
& \quad+\left\|u^{\cdot}(k+i \mid k)-u_{r e f}\right\|_{R}^{2}, \\
& V_{f}\left(x, r^{\bullet}, x_{r e f}\right)=\left\|x^{\bullet}(k+N \mid k)-x_{r e f}\right\|_{P}^{2} .
\end{aligned}
$$

Considering the state and input constraints, the MPC problem to be solved at each time step $k$ is

$$
\begin{array}{ll}
\min _{\boldsymbol{u}} & V_{N}\left(x^{\bullet}, r^{\bullet}, x_{\text {ref }}, u_{\text {ref }} ; \boldsymbol{u}\right) \\
\text { s.t. } & x^{\bullet}(0)=x^{\bullet}(k \mid k), \\
& x^{\bullet}(k+i \mid k) \\
& =A^{\bullet} x^{\bullet}(k+i-1 \mid k)+B_{u}^{\bullet} u^{\bullet}(k+i-1 \mid k) \\
& +B_{r}^{\bullet} r^{\bullet}(k+i-1 \mid k)+E^{\bullet}, \\
& u^{\bullet}(k+i \mid k) \in U, \\
& x^{\bullet}(k+i \mid k) \in X,
\end{array}
$$

in which the solution is the input trajectory $\boldsymbol{u}=\left\{\boldsymbol{u}^{\bullet}(k \mid\right.$ $\left.k), \ldots, u^{\bullet}(k+N-1 \mid k)\right\}$, and the first element of the sequence $u^{\bullet}(k \mid k)$ is applied to the plant. Figure 11 shows the results of this strategy under the scenario defined in Section 3.1.

A.2.2. Impulsive Zone MPC (iZMPC) [22]. The iZMPC is formulated in a similar manner to the corresponding discrete formulation by using a new decision variable $\delta$, which makes the cost zero when the predicted variables are inside the target zone and different from zero when they are outside it. The cost function is then given by

$$
\begin{aligned}
V_{N}\left(x^{\bullet}, r^{\bullet}, x_{r e f}, u_{r e f} ; \boldsymbol{u}, \delta\right) \\
=V_{d y n}\left(x^{\bullet}, r^{\bullet}, x_{r e f}, u_{r e f} ; \boldsymbol{u}, \delta\right) \\
\quad+V_{f}\left(\dot{x}^{\bullet}, r^{\bullet}, x_{r e f} ; \delta\right),
\end{aligned}
$$

where $V_{d y n}$ and $V_{f}$ are

$$
\begin{aligned}
& V_{d y n}\left(x^{\bullet}, r^{\bullet}, x_{r e f}, u_{r e f} ; \boldsymbol{u}, \delta\right) \\
& \quad=\sum_{i=0}^{N-1}\left\|x^{\cdot}(k+i \mid k)-x_{r e f}+\delta\right\|_{Q}^{2} \\
& \quad+\left\|u^{\cdot}(k+i \mid k)-u_{r e f}\right\|_{R}^{2}, \\
& V_{f}\left(x^{\cdot}, r^{\bullet}, x_{r e f} ; \delta\right)=\left\|x^{\cdot}(k+N \mid k)-x_{r e f}+\delta\right\|_{P}^{2} .
\end{aligned}
$$

The parameters $x_{r e f}$ and $u_{\text {ref }}$ are selected as a reference inside the target zone. Considering the state and input constraints, the MPC problem to be solved at each time step $k$ is

$$
\min _{\boldsymbol{u}, \delta} V_{N}\left(\dot{x}^{\bullet}, r^{\bullet}, x_{r e f}, u_{r e f} ; \boldsymbol{u}, \delta\right)
$$




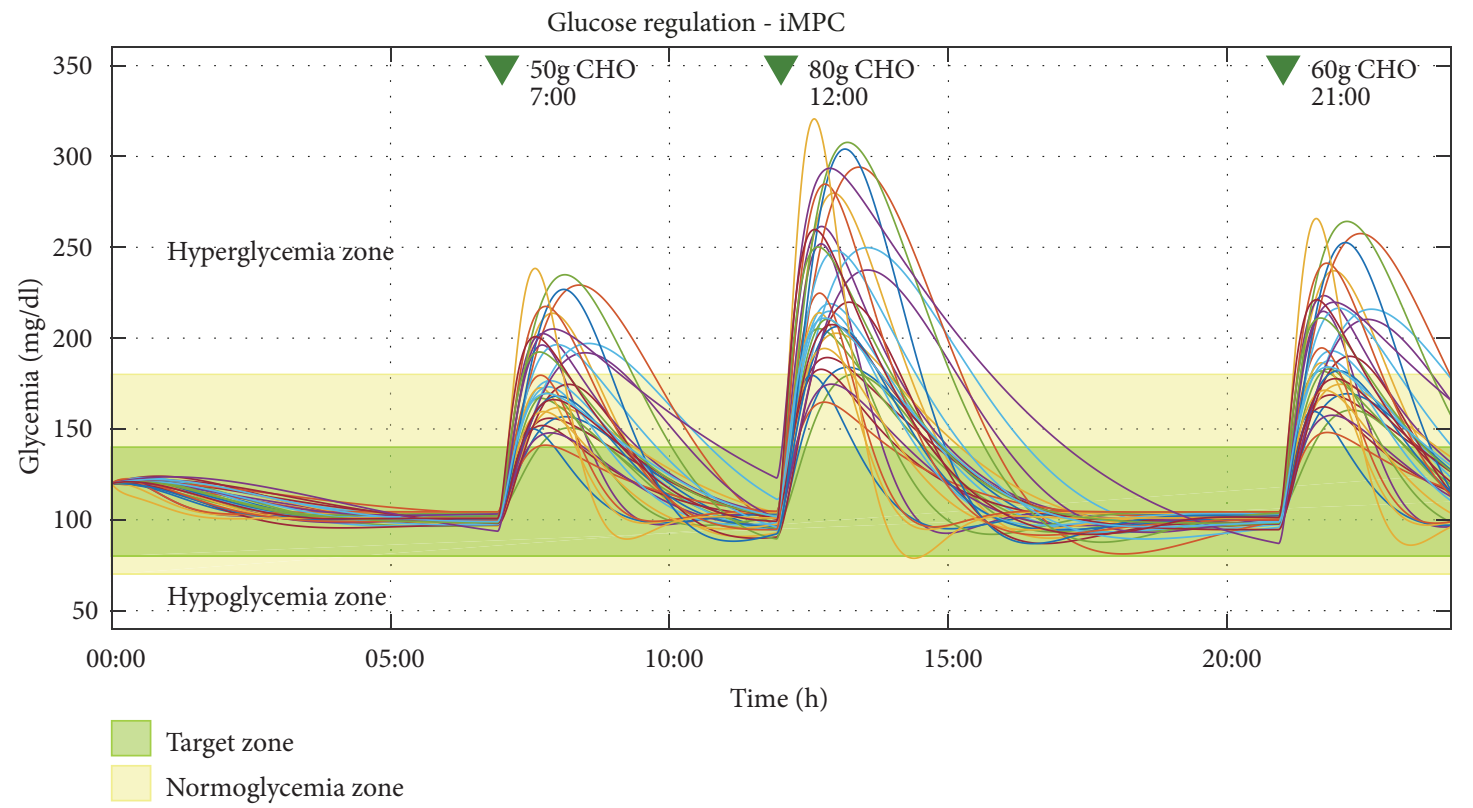

FIGURE 11: Blood glucose regulation for 33 virtual patients using the iMPC.

$$
\begin{array}{ll}
\text { s.t. } \quad & x^{\bullet}(0)=x^{\bullet}(k \mid k), \\
& x^{\bullet}(k+i \mid k) \\
= & A^{\bullet} x^{\bullet}(k+i-1 \mid k)+B_{u^{\bullet}}^{\cdot}(k+i-1 \mid k) \\
& +B_{r}^{\bullet} r^{\bullet}(k+i-1 \mid k)+E^{\bullet}, \\
& u^{\bullet}(k+i \mid k) \in U, \\
& x^{\bullet}(k+i \mid k) \in X, \\
& \delta_{\text {min }} \leq \delta \leq \delta_{\text {max }},
\end{array}
$$

in which the solution is the input trajectory $\boldsymbol{u}=\left\{\boldsymbol{u}^{\bullet}(k \mid\right.$ $\left.k), \ldots, u^{\bullet}(k+N-1 \mid k)\right\}$, and the first element of the sequence $u^{\bullet}(k \mid k)$ is applied to the plant. In Figure 12 its corresponding results are plotted.

A.2.3. Impulsive Zone MPC with Artificial Variables (iZMPC$A V)$ [10]. The formulation of this strategy is detailed in Section 2.2 , by using the artificial variables as decision variables. Figure 13 illustrates the performance of the control strategy which manages to lead the system to a target equilibrium set $X_{s}^{\cdot \text { Tar }} \subseteq X_{s}$.

Figures 8 and 11 present the glycemia behavior for the 33 patients using the standard MPC in its discrete and impulsive form. Though both controllers present similar performances, the dMPC manifests more excursions out of the target zone. Both controllers maintain all patients in the safe zone most of the time without risk of level 2 hyperglycemia or hypoglycemia episodes (see Table 2).

Figures 9 and 12 present the glycemia results for the patients for the ZMPC with slack variable, discrete and impulsive, respectively. The additional decision slack variable, $\delta$, allows the reduction of unnecessary doses of insulin when glycemia is in the target zone. Both controllers present postprandial excursions of hyperglycemia but achieve elimination of hypoglycemia events; i.e., glycemia of all the patients remains over $70 \mathrm{mg} / \mathrm{dl}$ (see Table 2 for more details).

Lastly, the comparison between dZMPC and iZMPC with artificial variables (dZMPC-AV and iZMPC-AV) is illustrated in Figures 10 and 13. For this formulation, the concept of reference is eliminated and the objective set is used as is described in the Methodology. Both controllers present postprandial hyperglycemia periods and zero periods of hypoglycemia. The time in the target zone is longer than the previous formulations, due to the equilibrium constraints. Both formulations present an adequate control in $100 \%$ of the cases (see Table 2).

\section{Data Availability}

The data used to support the findings of this study are available from the corresponding author upon request.

\section{Conflicts of Interest}

The authors declare that there are no conflicts of interest regarding the publication of this paper.

\section{Acknowledgments}

This research was supported by the Colciencias project number 110180763081 funded by the program "Convocatoria para Proyectos de Ciencia, Tecnología e Innovación en Salud 2018” from the Government of Colombia. 


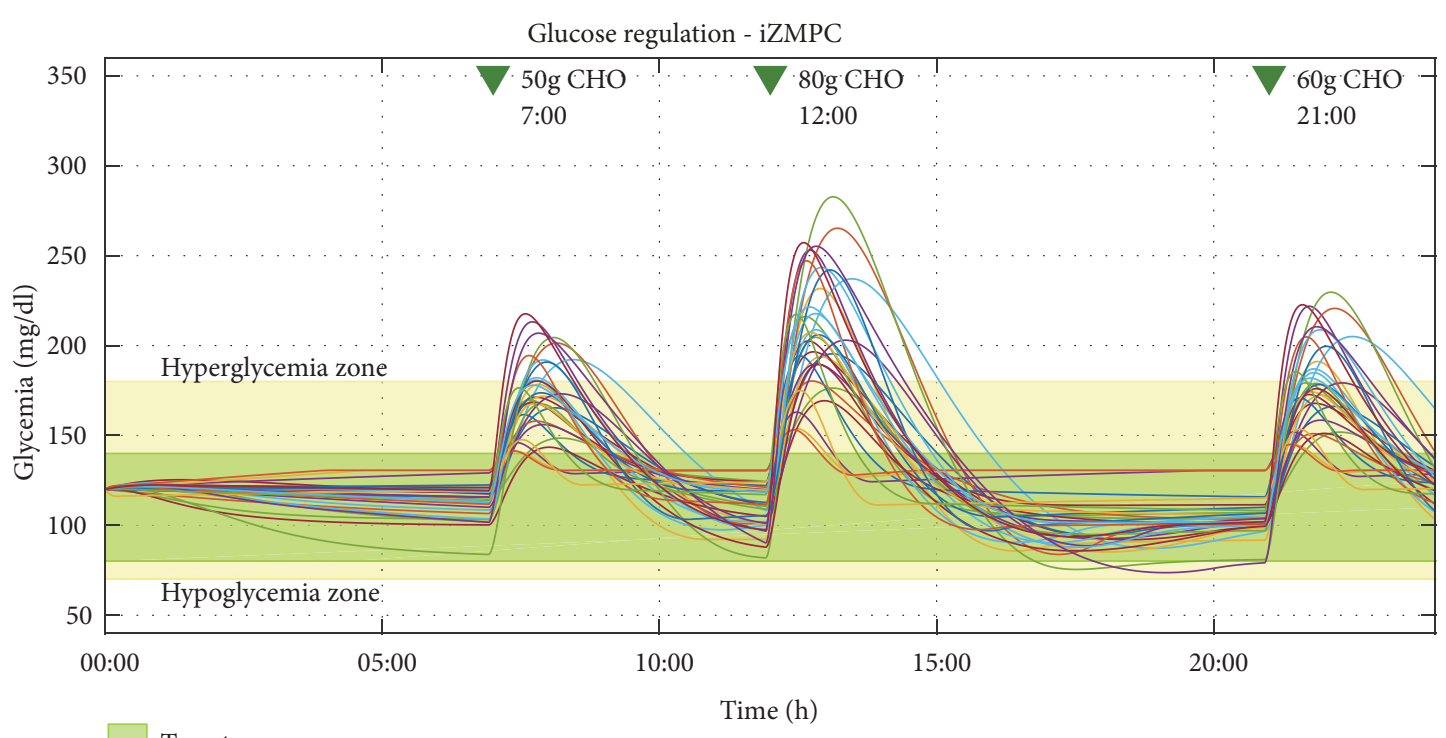

Target zone

Normoglycemia zone

FIGURE 12: Blood glucose regulation for 33 virtual patients using iZMPC.

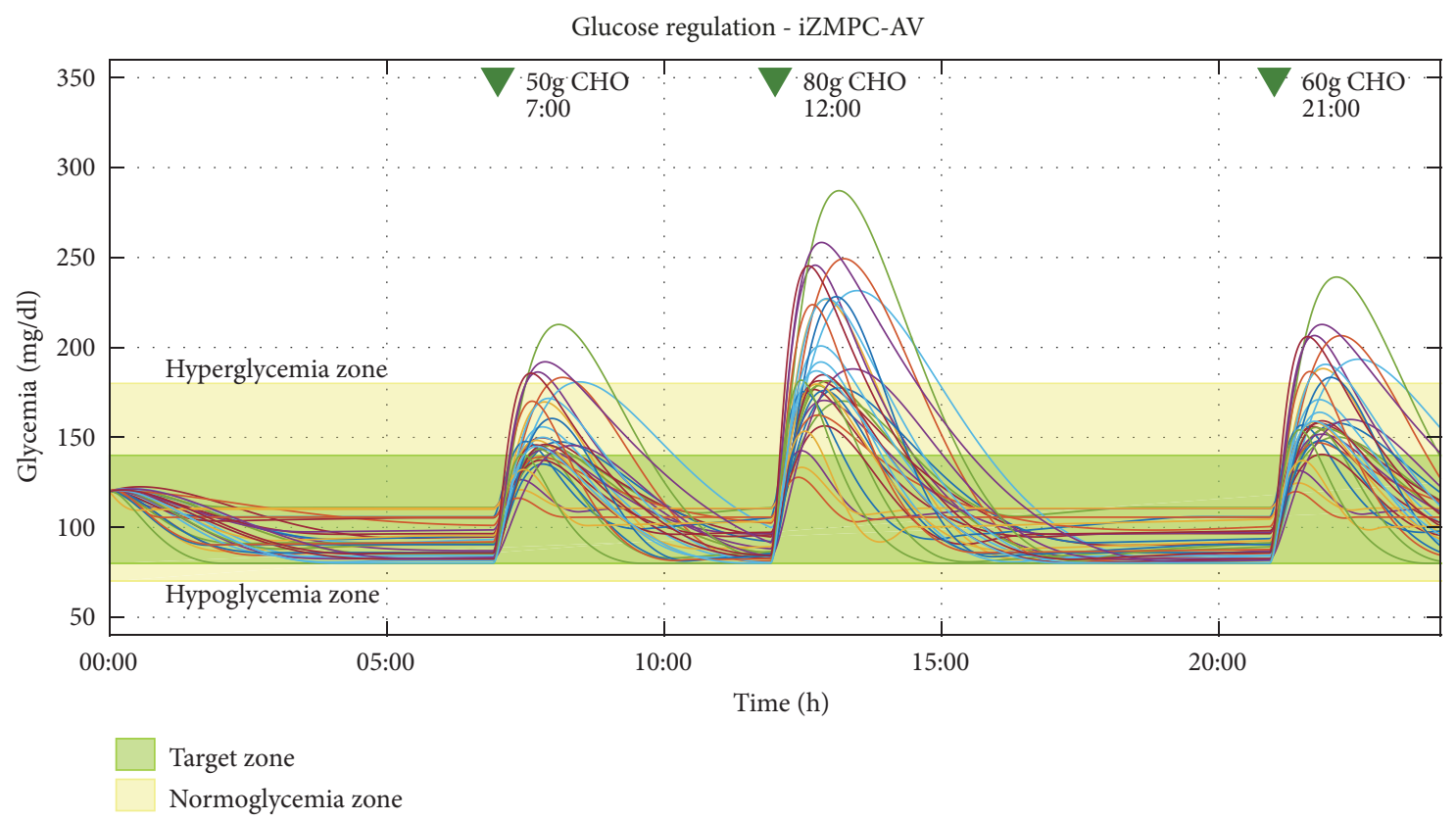

FIGURE 13: Blood glucose regulation for 33 virtual patients using the iZMPC-AV.

\section{References}

[1] C. p. Kasper, E. Braunwald, A. Fauci et al., Harrison's Principles of Internal Medicine, vol. 2, Mc Graw hill, 16 edition, 2006.

[2] M. Pombo, L. Audí, M. Bueno et al., Tratado De Endocrinología Pediátrica, McGraw-Hill Interamericana, Madrid, Spain, 4th edition, 2010.

[3] T. T. Ly, A. Roy, B. Grosman et al., "Day and night closedloop control using the integrated Medtronic hybrid closed-loop system in type 1 diabetes at diabetes camp," Diabetes Care, vol. 38, no. 7, pp. 1205-1211, 2015.
[4] J. E. Pinsker, J. B. Lee, E. Dassau et al., "Randomized crossover comparison of personalized MPC and PID control algorithms for the artificial pancreas," Diabetes Care, vol. 39, no. 7, pp. 1135$1142,2016$.

[5] E. Dassau, J. E. Pinsker, Y. C. Kudva et al., "12-week 24/7 ambulatory artificial pancreas with weekly adaptation of insulin delivery settings: effect on hemoglobin Alc and hypoglycemia," Diabetes Care, vol. 40, no. 12, pp. 1719-1726, 2017.

[6] S. Trevitt, S. Simpson, and A. Wood, "Artificial pancreas device systems for the closed-loop control of type 1 diabetes: what systems are in development?" Journal of Diabetes Science and Technology, vol. 10, no. 3, pp. 714-723, 2016. 
[7] B. Grosman, E. Dassau, H. C. Zisser, L. Jovanovič, and F. J. Doyle III, "Zone model predictive control: A strategy to minimize hyper- and hypoglycemic events," Journal of Diabetes Science and Technology, vol. 4, no. 4, pp. 961-975, 2010.

[8] P. S. Rivadeneira and C. H. Moog, "Observability criteria for impulsive control systems with applications to biomedical engineering processes," Automatica, vol. 55, pp. 125-131, 2015.

[9] P. S. Rivadeneira, A. Ferramosca, and A. H. González, "Impulsive zone model predictive control with application to Type I Diabetic Patients," in Proceedings of the IEEE Conference on Control Applications, pp. 544-549, 2016.

[10] P. S. Rivadeneira, A. Ferramosca, and A. H. González, “Control strategies for nonzero set-point regulation of linear impulsive systems," IEEE Transactions on Automatic Control, vol. 63, no. 9, pp. 2994-3001, 2018.

[11] R. N. Bergman, L. S. Phillips, and C. Cobelli, "Physiologic evaluation of factors controlling glucose tolerance in man. Measurement of insulin sensitivity and $\beta$-cell glucose sensitivity from the response to intravenous glucose," The American Society for Clinical Investigation, vol. 68, no. 6, pp. 1456-1467, 1981.

[12] C. Dalla Man, R. A. Rizza, and C. Cobelli, "Meal simulation model of the glucose-insulin system," IEEE Transactions on Biomedical Engineering, vol. 54, no. 10, pp. 1740-1749, 2007.

[13] C. Dalla Man, F. Micheletto, D. Lv, M. Breton, B. Kovatchev, and C. Cobelli, "The UVA/PADOVA type 1 diabetes simulator: New features," Journal of Diabetes Science and Technology, vol. 8, no. 1, pp. 26-34, 2014.

[14] C. Toffanin, M. Messori, F. Di Palma, G. De Nicolao, C. Cobelli, and L. Magni, "Artificial pancreas: Model predictive control design from clinical experience," Journal of Diabetes Science and Technology, vol. 7, no. 6, pp. 1470-1483, 2013.

[15] N. Magdelaine, L. Chaillous, I. Guilhem et al., "A long-term model of the glucose-insulin dynamics of type 1 diabetes," IEEE Transactions on Biomedical Engineering, vol. 62, no. 6, pp. 15461552, 2015.

[16] Y. Ruan, M. E. Wilinska, H. Thabit, and R. Hovorka, "Modelling day-to-day variability of glucose-insulin regulation over 12week home use of closed-loop insulin delivery," IEEE Transactions on Biomedical Engineering, 2016.

[17] B. Grosman, D. Wu, D. Miller et al., "Sensor-augmented pumpbased customized mathematical model for type 1 diabetes," Diabetes Technology \& Therapeutics, vol. 20, no. 3, pp. 207-221, 2018.

[18] J. D. Hoyos, F. Bolanos, M. Vallejo, and P. S. Rivadeneira, "Population-based incremental learning algorithm for identification of blood glucose dynamics model for type-1 diabetic patients," in Proceedings of the International Conference on Artificial Intelligence, pp. 29-35, 2018.

[19] H. K. Khalil, Nonlinear Systems, Prentice Hall, New Jersey, USA, 2nd edition, 1996.

[20] U. Maeder, F. Borrelli, and M. Morari, "Linear offset-free model predictive control," Automatica, vol. 45, no. 10, pp. 2214-2222, 2009.

[21] G. Pannocchia, "Offset-free tracking MPC: A tutorial review and comparison of different formulations," in Proceedings of the European Control Conference, pp. 527-532, 2015.

[22] P. S. Rivadeneira, J. E. Sereno, and M. A. Caicedo, "Nuevas estrategias de control glucémico en pacientes con diabetes mellitus tipo 1," Revista Iberoamericana de Automática e Informàtica Industrial, vol. 16, pp. 238-248, 2019.
[23] P. S. Rivadeneira, A. Ferramosca, and A. H. González, "MPC with state window target control in linear impulsive systems," IFAC-PapersOnLine, vol. 48, no. 23, pp. 507-512, 2015.

[24] J. Maciejowski, Predictive Control with Constraints, Prentice Hall, London, UK, 2002.

[25] J. B. Rawlings and D. Q. Mayne, Model Predictive Control: Theory and Design, Nob hill Publishing, Wis, USA, 2015.

[26] T. J. van den Boom and A. A. Stoorvogel, "Model Predictive Control," disc, 2010.

[27] D. Simon, Optimal State Estimation: Kalman, H Infinity, and Nonlinear Approaches, Wiley Interscience, New Jersey, USA, 2006.

[28] A. Ferramosca, A. H. González, D. Odloak, and E. F. Camacho, "MPC for tracking target sets," in Proceedings of the 48h IEEE Conference on Decision and Control held jointly with 2009 28th Chinese Control Conference, pp. 8020-8025, 2009.

[29] A. Ferramosca, D. Limon, A. H. González, D. Odloak, and E. F. Camacho, "MPC for tracking zone regions," Journal of Process Control, vol. 20, no. 4, pp. 506-516, 2010.

[30] P. Sopasakis, P. Patrinos, H. Sarimveis, and A. Bemporad, "Model predictive control for linear impulsive systems," Institute of Electrical and Electronics Engineers Transactions on Automatic Control, vol. 60, no. 8, pp. 2277-2282, 2015.

[31] J. E. Sereno and P. S. Rivadeneira, "Auto-tuning for model predictive controllers in patients with type 1 diabetes," in Proceedings of the 2018 Argentine Conference on Automatic Control, 2018.

[32] P. Soru, G. De Nicolao, C. Toffanin, C. Dalla Man, C. Cobelli, and L. Magni, "MPC based Artificial Pancreas: Strategies for individualization and meal compensation," Annual Reviews in Control, vol. 36, no. 1, pp. 118-128, 2012.

[33] T. Danne, R. Nimri, T. Battelino et al., "International consensus on use of continuous glucose monitoring," Diabetes Care, vol. 40, no. 12, pp. 1631-1640, 2017.

[34] D. M. Maahs, B. A. Buckingham, J. R. Castle et al., "Outcome measures for artificial pancreas clinical trials: A consensus report," Diabetes Care, vol. 39, no. 7, pp. 1175-1179, 2016.

[35] A. El Fathi, M. R. Smaoui, V. Gingras, B. Boulet, and A. Haidar, "The artificial pancreas and meal control: an overview of postprandial glucose regulation in type 1 diabetes," IEEE Control Systems Magazine, vol. 38, no. 1, pp. 67-85, 2018.

[36] L. Magni, D. M. Raimondo, C. Dalla Man et al., "Evaluating the efficacy of closed-loop glucose regulation via control-variability grid analysis," Journal of Diabetes Science and Technology, vol. 2, no. 4, pp. 630-635, 2008.

[37] M. Breton and B. Kovatchev, "Analysis, modeling, and simulation of the accuracy of Continuous glucose sensors," Journal of Diabetes Science and Technology, vol. 2, no. 5, pp. 853-862, 2008. 


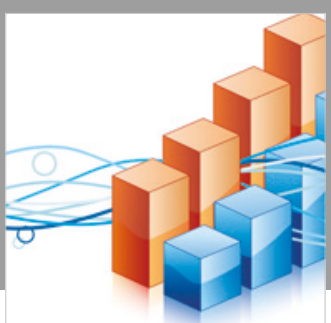

Advances in

Operations Research

\section{-n-m}
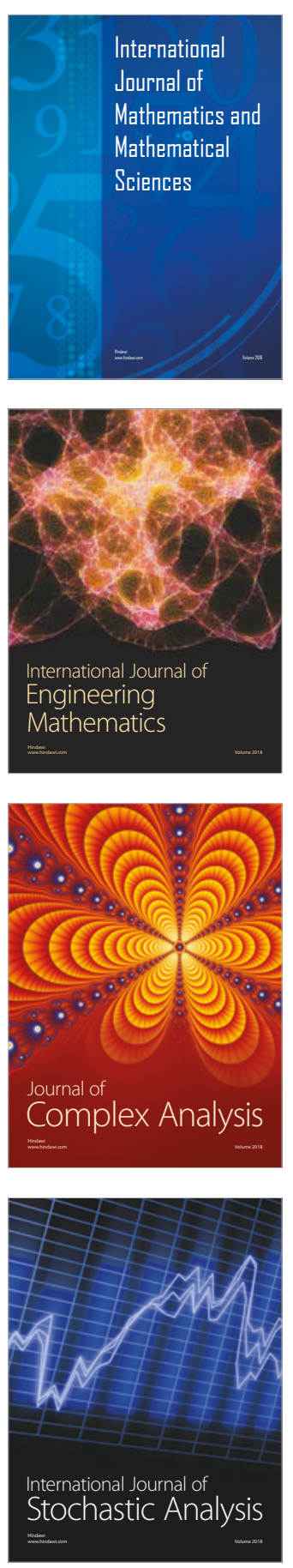
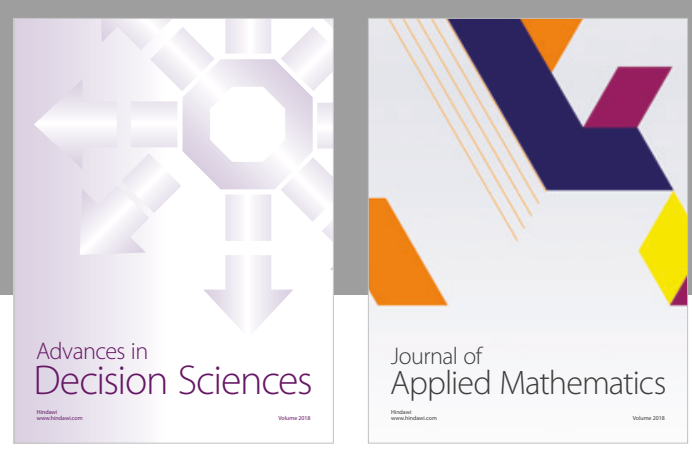

Journal of

Applied Mathematics
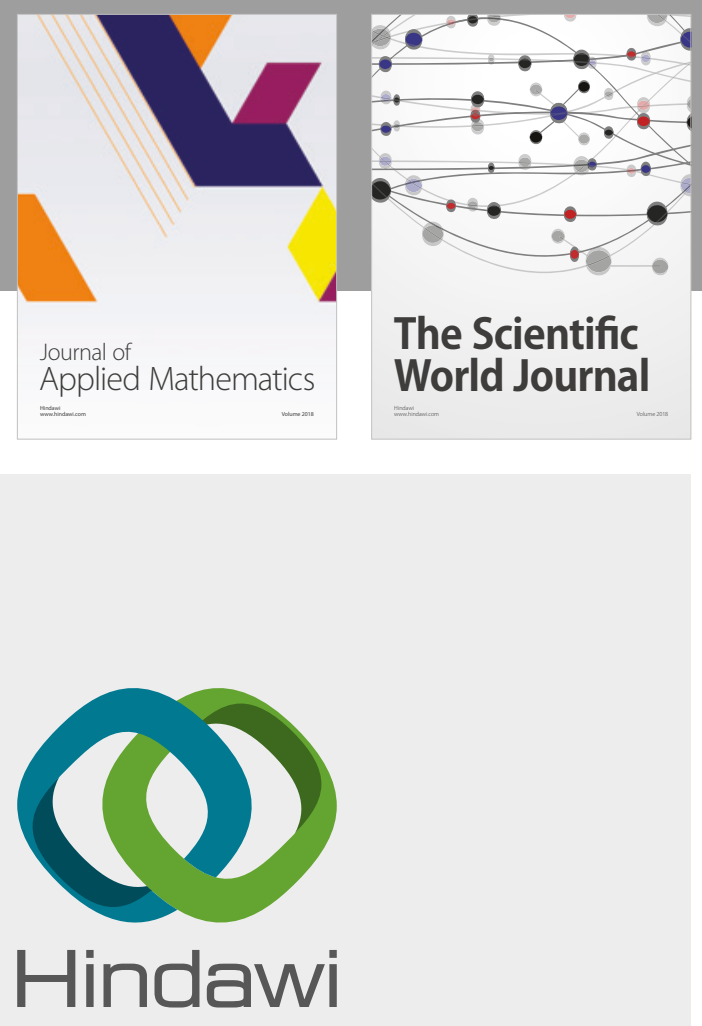

Submit your manuscripts at

www.hindawi.com

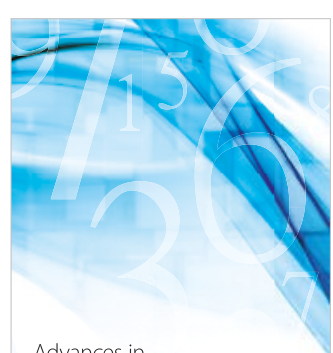

Advances in
Numerical Analysis
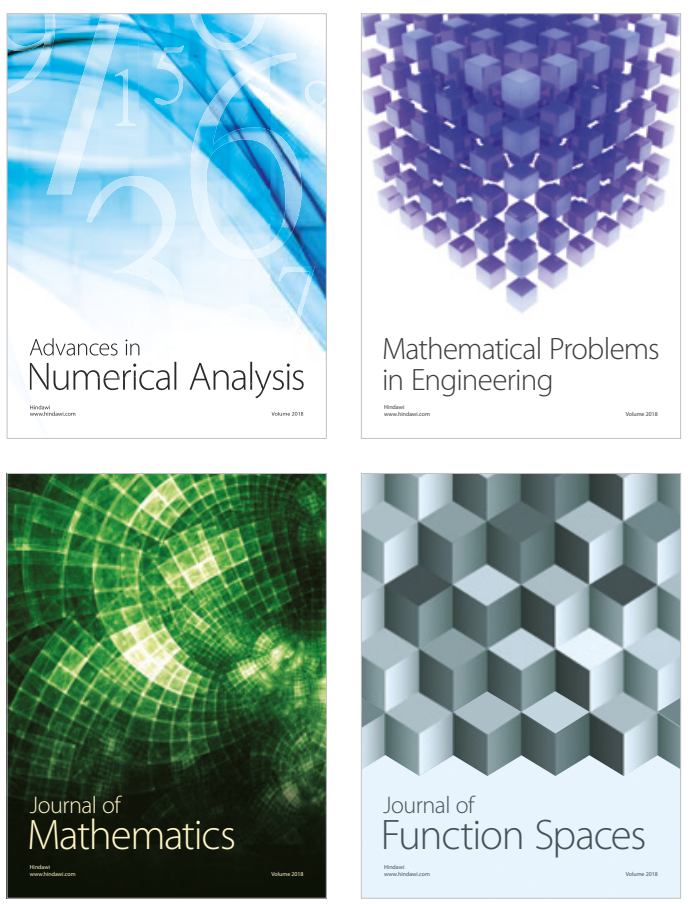

Mathematical Problems in Engineering

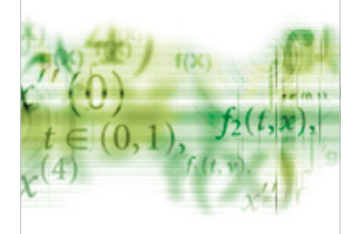

International Journal of

Differential Equations

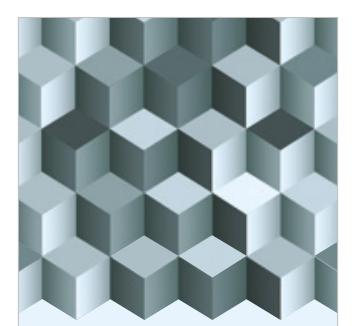

Journal of

Function Spaces

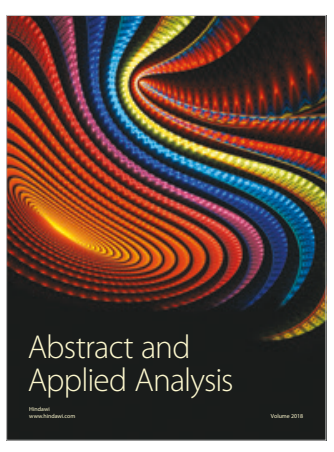

The Scientific

World Journal

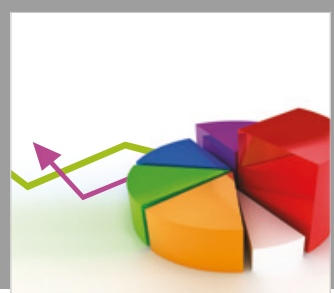

Journal of

Probability and Statistics
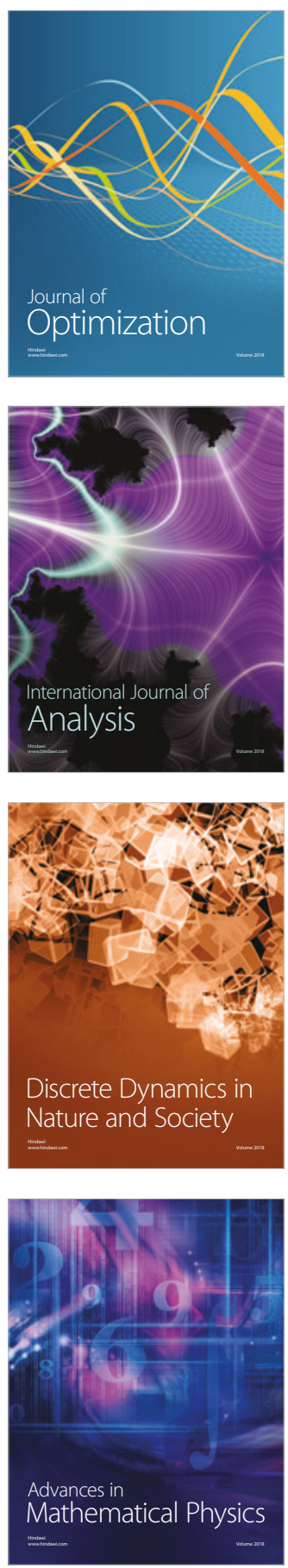